\title{
Hyper-Heuristics based on Reinforcement Learning, Balanced Heuristic Selection and Group Decision Acceptance
}

\author{
Valdivino Alexandre de Santiago Júnior ${ }^{\mathrm{a}, \mathrm{b}, *}$, Ender Özcan $^{\mathrm{b}}$, Vinicius Renan de Carvalho ${ }^{\mathrm{c}, \mathrm{b}}$ \\ ${ }^{a}$ Instituto Nacional de Pesquisas Espaciais (INPE) \\ Laboratório Associado de Computação e Matemática Aplicada (LABAC) - Av. dos Astronautas, 1758, São José \\ dos Campos, SP, Brazil, 12227-010 \\ ${ }^{b}$ University of Nottingham, \\ Computational Optimisation and Learning (COL) Lab, School of Computer Science, Wollaton Road, \\ Nottingham, United Kingdom, NG8 $1 B B$ \\ ${ }^{c}$ Universidade de São Paulo (USP) \\ Escola Politécnica (Poli) - Av. Prof. Luciano Gualberto, 380, São Paulo, SP, Brazil, 05508-010
}

\begin{abstract}
In this paper, we introduce a multi-objective selection hyper-heuristic approach combining Reinforcement Learning, (meta)heuristic selection, and group decision-making as acceptance methods, referred to as Hyper-Heuristic based on Reinforcement LearnIng, Balanced Heuristic Selection and Group Decision AccEptance (HRISE), controlling a set of Multi-Objective Evolutionary Algorithms (MOEAs) as Low-Level (meta)Heuristics (LLHs). Along with the use of multiple MOEAs, we believe that having a robust LLH selection method as well as several move acceptance methods at our disposal would lead to an improved general-purpose method producing most adequate solutions to the problem instances across multiple domains. We present two learning hyperheuristics based on the HRISE framework for multi-objective optimisation, each embedding a group decision-making acceptance method under a different rule: majority rule (HRISE_M) and responsibility rule (HRISE_R). A third hyper-heuristic is also defined where both a random LLH selection and a random move acceptance strategy are used. We also propose two variants of the late acceptance method and a new quality indicator supporting the initialisation of selection hyperheuristics using low computational budget. An extensive set of experiments were performed using 39 multi-objective problem instances from various domains where 24 are from four different benchmark function classes, and the remaining 15 instances are from four different real-world problems. The cross-domain search performance of the proposed learning hyper-heuristics indeed turned out to be the best, particularly HRISE_R, when compared to three other selection hyper-heuristics, including a recently proposed one, and all low-level MOEAs each run in isolation.
\end{abstract}

Keywords: Hyper-Heuristic, Reinforcement Learning, Balanced Heuristic Selection, Group Decision-Making, Multi-Objective Evolutionary Algorithms, Multi-Objective Optimisation

\footnotetext{
* Corresponding author

Email addresses: valdivino.santiago@inpe.br (Valdivino Alexandre de Santiago Júnior), Ender.Ozcan@nottingham.ac.uk (Ender Özcan), vrcarvalho@usp.br (Vinicius Renan de Carvalho)
} 


\section{Introduction}

The main motivation behind hyper-heuristics is to resolve some of the issues with metaheuristics. Although metaheuristics have long been proved beneficial to solve real-world complex search problems such as scheduling, clustering, educational timetabling, and space allocation, it is still not

5 straightforward to apply them directly to new optimisation problems from different domains with no or minimal change, or even to new instances of the same problem [1, 2]. Some of the reasons of such difficulties are the usual high number of parameters or algorithm choices the practitioner must define, and the absence of proper guidelines to select them.

Hence, hyper-heuristics have emerged which represent a class of high-level search techniques whose goal is to raise the level of generality at which search methods work [1, 2, In hyperheuristics, the search is performed in the space of heuristics (or heuristics components) instead of being performed directly in the decision variable space (space of solutions). Thus, hyper-heuristics are more general and so appropriate to solve different problems rather than a specific problem based on a set of Low-Level (meta)Heuristics (LLHs).

A generic selection hyper-heuristic consists of two key components: heuristic selection and move acceptance methods [3]. As the name implies, in heuristic selection there is a set of LLHs and the strategy must choose the most appropriate one to run at a certain moment of the search process. The move acceptance method decides whether a solution (or a subset of solutions) that is the result of the execution of the selected LLH is accepted or not. As we will explain below, most studies do not exploit the benefits of relying on a robust heuristic selection strategy while simultaneously having a more elaborate and effective move acceptance method for accepting the generated solutions (e.g. in a population).

There are many studies on selection hyper-heuristics based on perturbative LLHs mostly using a single point-based search framework for single objective optimisation [3, 4, 5, 6, 17, 8, However, the use of population-based methods is a recently growing area of research [9] where hyper-heuristics for multi-objective optimisation are employed either for controlling the own components of MultiObjective Evolutionary Algorithms (MOEAs) [10, 11, 12, e.g. mutation and crossover operators as LLHs, or the LLHs being complete MOEAs [13, 14, 15, 16].

Despite these efforts and even if there are several proposed selection of LLHs and move acceptance methods, the hyper-heuristics usually consist of a single LLH selection method, with no additional policies, combined with a single move acceptance approach. Few studies have made a contribution where more than one heuristic selection/policy and/or move acceptance method is considered. For instance, Li et al. (2019) [16] proposed a new heuristic selection method, $\epsilon$ RouletteGreedy, which is based on greedy and roulette wheel strategies. But only a single move 
35 acceptance method is used. In Kheiri et al. (2016) [17, ensemble move acceptance methods are combined under a group decision-making framework but employing a single point-based search.

We believe that having a set of LLH selection, policies, and move acceptance methods at our disposal can produce a most adequate strategy to solve better the optimisation problems, since we can benefit of the combined strengths of all the techniques. Thus, in this paper we present a novel online selection hyper-heuristic based on perturbative LLHs which aims to solve complex multi-objective problems via a heuristic selection approach based on Reinforcement Learning and a balanced mechanism, and move acceptance methods. The Hyper-Heuristic based on Reinforcement LearnIng, Balanced Heuristic Selection and Group Decision AccEptance (HRISE) contemplates a single LLH selection method but with additional policies and three move acceptance methods within group decision-making rules. Our hyper-heuristic considers as LLHs complete MOEAs and we indeed propose two variants of it based on group decision-making acceptance: one based on the majority rule (HRISE_M) and another one based on the responsibility rule (HRISE_R). A third hyper-heuristic, called Hyper-Heuristic based on Random LLH Selection and Random Choice of Move Acceptance Methods (HRMA) is also proposed where both a random choice of (meta)heuristic selection is performed combined with a random choice of move acceptance methods.

We compared the performance of HRISE_M, HRISE_R, and HRMA to three other hyperheuristics, including the Choice Function hyper-heuristic (HH-CF) [13], a random choice (meta)heuristic selection hyper-heuristic with All Moves acceptance (HH-ALL), and a recent one Learning Automatabased Hyper-Heuristic with a Ranking Scheme Initialisation (HH-RILA) [16 which is the stateof-the-art. We also performed the comparison to the following three MOEAs run in isolation which are the LLHs in all hyper-heuristics: Nondominated Sorting Genetic Algorithm-II (NSGAII) [18, Indicator-Based Evolutionary Algorithm (IBEA) [19], and Strength Pareto Evolutionary Algorithm-2 (SPEA2) [20].

The main contributions of this study are:

1. We propose two new selection hyper-heuristics (HRISE_M and HRISE_R) based on an approach embedding a robust heuristic selection strategy, via Reinforcement Learning, and enabling the use of several move acceptance methods under a group-decision framework. Our idea is to rely on a range of methods not only to select a new LLH but also to accept a population of trade-off solutions generated after the execution of a selected LLH. To the best of our knowledge, no previous approach has stressed both the heuristic selection (roulette wheel supported by Reinforcement Learning plus a balanced exploitation/exploration method) and the move acceptance (two-level strategy: Only Improving + group decision-making) mechanisms as we suggest in this work;

2. We propose two new move acceptance methods based on Late Acceptance [21, 22]. In 


\section{Related Work}

In this section we present some relevant studies related to selection hyper-heuristics based on perturbative LLHs. We divide them in three categories: single point search-based, populationbased with components of MOEAs, and population-based with complete MOEAs.

95 in [2. The authors distributed the studies in several classes such as hyper-heuristics using deterministic move acceptance [4, 5], hyper-heuristics using heuristic selection with no learning and non-deterministic move acceptance [6], and hyper-heuristics using heuristic selection with online learning and non-deterministic move acceptance [3, 7, 8. Although there are several heuristic selection of LLH method with no additional policies, combined with a single move acceptance 
approach. In this study, we use roulette wheel supported by Reinforcement Learning together with policies for selecting LLHs, and a two-level (hierarchical) move acceptance method formed by Only Improving and group decision acceptance.

With respect to the studies of selection hyper-heuristics within population-based approaches, here we see strategies which control components (e.g. crossover and mutation operators) of a MOEA [9]. In [23, the Water Distribution Network (WDN) design problem is addressed via a selection hyper-heuristic based on Markov Chains [24] which is incorporated into NSGA-II and SPEA2. They considered a range of mutation and crossover operator-based LLHs. Some of these previous studies are in the context of Search-Based Software Testing (SBST) 25, 26, 27, 28, addressing problems such as integration and test order [10], derivation of products for Software Product Line (SPL) testing [11, and Second Order Mutants (SOMs) generation strategies [12]. All these studies only consider a single heuristic selection method (e.g. Choice Function, Upper Confidence Bound, Multi-Armed Bandit [29, 30]) with no additional policies to improve the overall approach. Moreover, we believe that if complete MOEAs are used as LLHs, we can benefit of the strengths of each low-level metaheuristic as a whole.

Recent studies fall then into this latter category where instead of operators of a single metaheuristic, complete MOEAs are used as LLHs. In [13], the authors presented a hyper-heuristic based on Choice Function in which NSGA-II, SPEA2, and the Multi-Objective Genetic Algorithm (MOGA) [31] are the LLHs. In [14, researchers used NSGA-II, IBEA, and SPEA2 as LLHs and evaluated several heuristic selection (Fixed Sequence, Choice Function) and acceptance (Great Deluge Acceptance (GDA) [3, 32] with D-metric, Best Acceptance) methods for wind farm layout optimisation. The same previous remarks apply here: only one heuristic selection, no additional policies, and only one acceptance method is defined for each hyper-heuristic.

MOABHH is an agent-based hyper-heuristic framework focused on online selection by means of voting techniques [15]. The main idea is to consider MOEAs as candidates and quality indicators as voters in an election. NSGA-II, SPEA2, IBEA, and the Generalised Differential Evolution 3 (GDE3) 33] are the LLHs. They used a voting method to select an LLH based on the Condorcet's principle which is similar to the group decision-making majority rule, but we used this rule as a move acceptor and not as a selector. Moreover, they used only the All Moves acceptance method while we have a set of acceptance methods.

In [16, the authors proposed a learning automata-based selection hyper-heuristic. There are two variants of the hyper-heuristic depending on whether a uniform (HH-LA) or a ranking-based (HH-RILA) initialisation process is chosen. LLHs are NSGA-II, IBEA, and SPEA2. They defined 135 a selection method, named $\epsilon$-RouletteGreedy, in which they apply roulette wheel in the initial stage and, after that, they select between greedy and roulette wheel based on $\epsilon$, the probability of applying the greedy heuristic selection method. Such a probability is increased linearly during 
the execution. In our case, we defined a light (low demanding in computational terms) initialisation process based on a new quality indicator, and our additional policies are within a robust balanced exploitation/exploration approach. They used only one move acceptance method (Only Improving) while we have a two-level (hierarchical) approach and, moreover, we also defined a nonuniform iterations strategy addressing the number of times an LLH can execute.

\section{The HRISE/HRMA Selection Hyper-Heuristics}

In this section, we describe both variants of the HRISE hyper-heuristic: HRISE_M and HRISE_R. We designed the HRMA hyper-heuristic under the same general structure of HRISE and hence we address it here too. Figure 1 presents our hyper-heuristics using an activity diagram while Algorithm 1 is the main HRISE/HRMA procedure where we show the main features of our proposals in an algorithmic format.

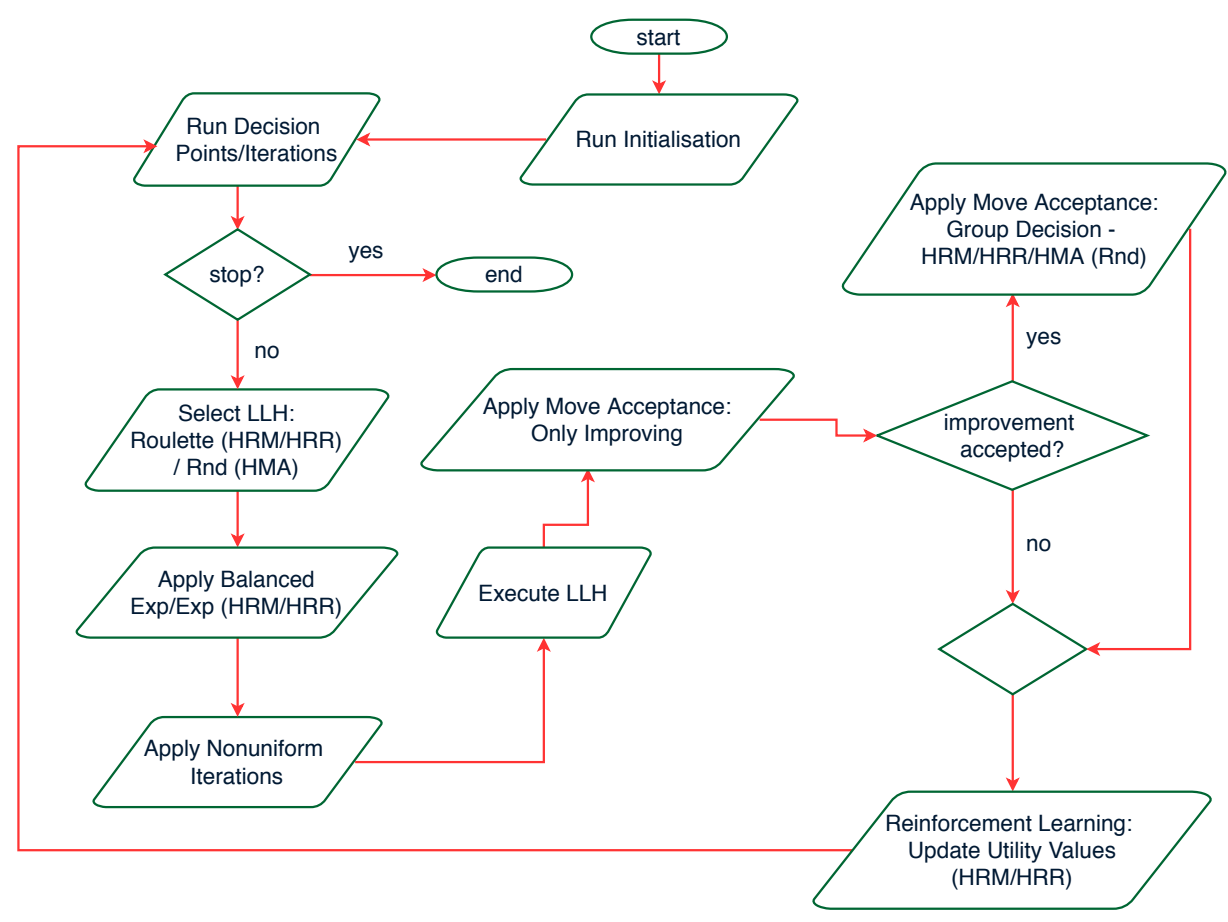

Figure 1: The HRISE/HRMA selection hyper-heuristics: activity diagram. Caption: HRM = HRISE_M; HRR = HRISE_R; HMA = HRMA; Rnd = Random; Exp/Exp = Exploitation/Exploration

We mention some general observations below to show the main differences between the three approaches. An in-depth explanation of the features of our hyper-heuristics are provided later in this section. The LLHs of HRISE are complete MOEAs hence we consider not only their components (e.g. mutation and crossover operations). Note that in Figure 1 some activities/tasks only exist for HRISE_M and HRISE_R, i.e. balanced exploitation/exploration (Apply Balanced Exp/Exp) and the activity related to Reinforcement Learning. Moreover, the heuristic selection is 
roulette wheel [34 for HRISE_M and HRISE_R and it is a random LLH selection within HRMA. In the group decision move acceptance mechanism, the majority (HRISE_M) or responsibility (HRISE_R) rules are used depending on the hyper-heuristic and, for HRMA, we have a random choice among the available move acceptance methods.

As shown in Algorithm 1 HRISE receives as inputs the problem instance (Pr), the version of the hyper-heuristic ( $v=M, R, A$ for HRISE_M, HRISE_R, HRMA, respectively), the maximum number of interations $(m i)$, the maximum number of decision points $(m d)$, the population size $(z)$, and the sequence $\varrho^{1}$ of LLHs $(L L H)$. The final resulting population is $P_{o p}{ }^{f}$.

The first activity in Figure 1 is the initialisation where we designed a computationally low demanding procedure (compLowInitialisationRPO in Algorithm 1) in which we run each LLH $k$ for very few iterations $\mathrm{L}^{2}$ and only once, and decide the first to be executed based on a new quality indicator which we detail in Section 3.1. We record the previous performance of an LLH $k$ in Per, a sequence whose elements indicate if $k$ had its offspring population accepted in the last time it was executed.

After the execution of the first selected LLH, for HRISE_M and HRISE_R, our approach first selects the next LLH to run based on roulette wheel 34] considering the utility values $(U V)$ associated with the LLHs. The utility value of an LLH $k, \mu_{k}$, influences the probability of $k$ to be selected and as higher the utility value, the better. After the first decision point, all LLHs have the same utility value and hence a random choice is made. However, the Reinforcement Learning step of our approaches (see Section 3.3) rewards or penalises an LLH according to its performance, and this reflects on the heuristic selection at future decision points.

As for HRISE_M and HRISE_R, a balanced exploitation/exploration procedure (balancedExpExp in Algorithm 1 more details in Section 3.2 is performed following the LLH selection via roulette wheel. As we have previously mentioned, for HRMA an LHH is randomly selected and neither the balanced exploitation/exploration mechanism nor the Reinforcement Learning step are used.

Traditionally, the number of iterations a selected LLH will run is fixed, e.g. an LLH runs for 10 iterations [16], 250 iterations [13, and so on. We can denote it as a uniform approach regarding the number of iterations/LLH.

But, we have observed that a nonuniform technique is worth being considered where the number of iterations an LLH will execute varies (nonUniformIterations in Algorithm 1). We envisage three scenarios as presented in Figure 2. The descending approach is where we give more iterations to a selected LLH to be executed within the very first decision points while providing less iterations

\footnotetext{
${ }^{1} \mathrm{~A}$ sequence differs from a set because repetition of elements is allowed and order matters. Even if we do not expect that the user inputs repeated names of metaheuristics, the order that they are defined is relevant because of the index, which is related to the metaheuristic, being used by other sequences of the approach.

${ }^{2}$ We prefer denoting "iteration" rather than "generation" in order to be more general. On the other hand, we use "decision point" in the same sense as other authors use "iteration".
} 


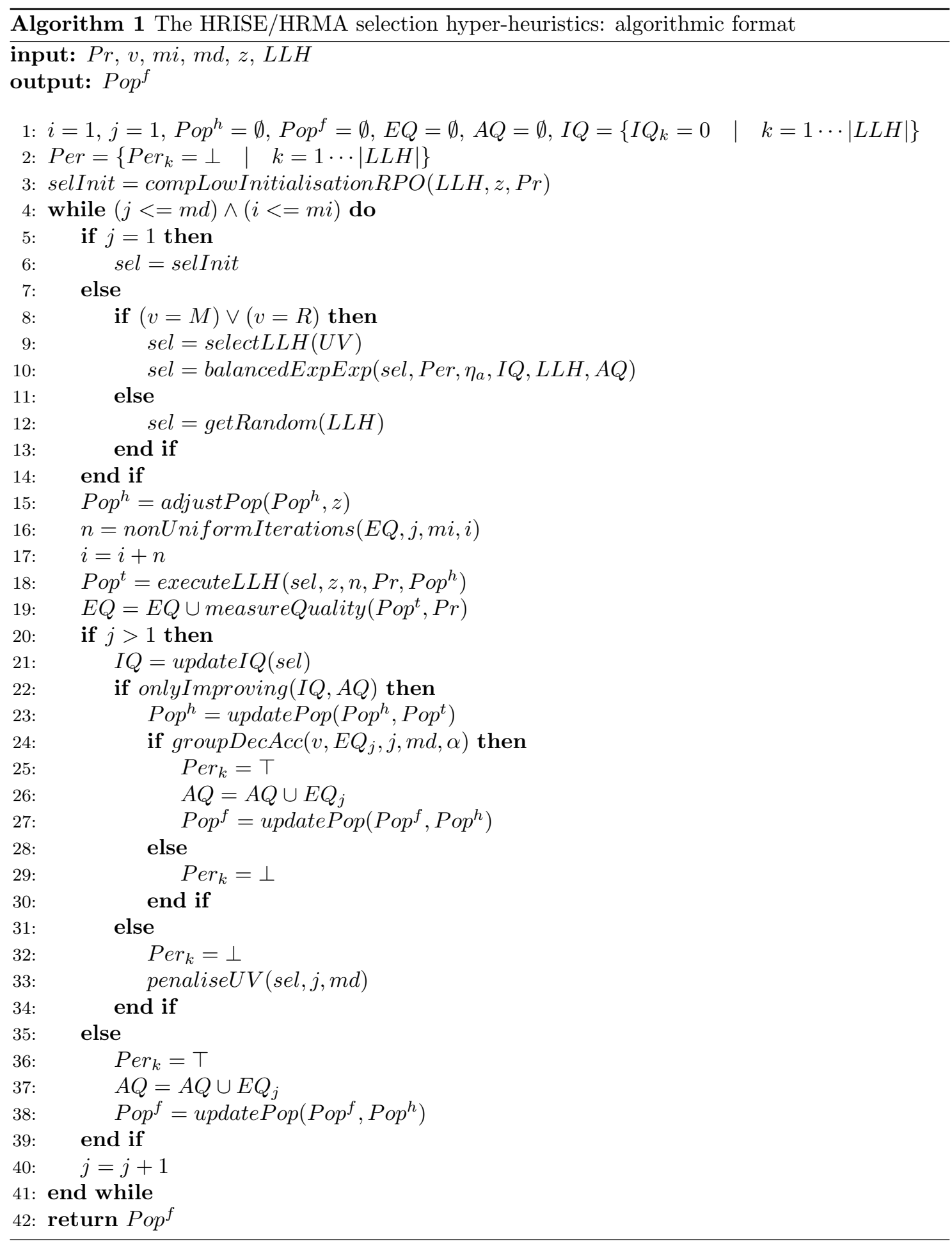


in the last decision points. The main reasoning behind this idea is trying to get fitter populations at the beginning by giving the first selected LLHs more iterations to run, but allowing more decision points to exist by providing less iterations later.
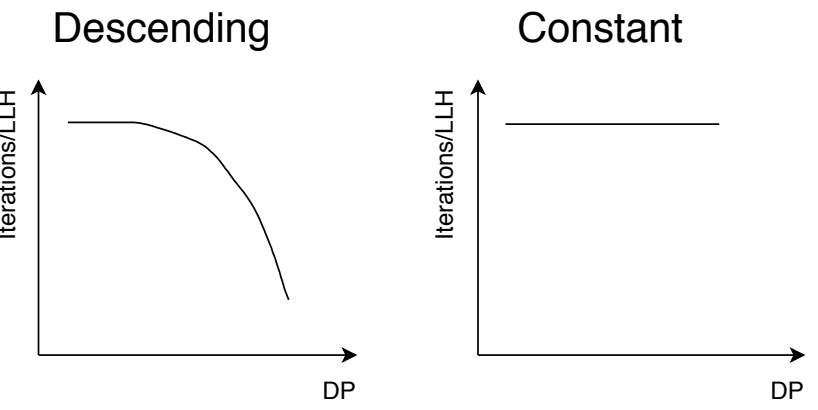

\section{Bathtub}

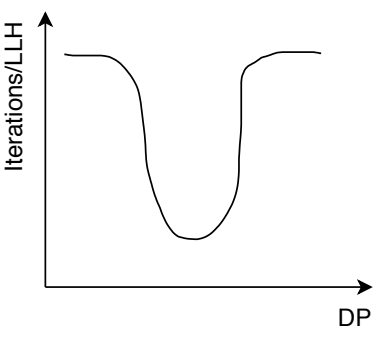

Figure 2: The nonuniform iterations strategy. Caption: $\mathrm{DP}=$ Decision Point

Even if we give a higher number of iterations at the beginning, it is possible, for some problem instances, that the populations consist of nondominated solutions but some values of objective functions are extremely high while others are very low. This may cause the hypervolume [35], the quality indicator we consider to drive decisions within our hyper-heuristics, to be extremely low or even 0 at the end of the number of iterations. Hence, by naturally decreasing the number of iterations as in the descending approach is not enough. In addition, we chose hypervolume as our key quality indicator since previous studies state that as maximum the hypervolume indicator is the better, because this is equivalent to optimising the overall objective leading to an optimal approximation of the True Pareto Front 36.

The constant strategy is then the one where we identify this issue and maintain the number of iterations huge and with the same value up to the termination criterion. The sequence $E Q$ is input to the nonuniform technique and has every value of quality indicators (hypervolumes) due to the offspring populations $\left(P o p_{t}\right)$. In this case, we check whether such hypervolume, $h_{j}$, is less than or equal to a threshold, $\gamma$, and count the number of these very low values. If this count exceeds 1 , hence we can conclude that it is important to keep constant and high the number of iterations/LLH.

The third scenario is called bathtub. In other words, we start with a high number of iterations, decrease this number of iterations as in the descending strategy but, at some point, we realise that offspring populations have not been accepted and hence we raise again the number of iterations and thus provide more resources for the later selected LLHs.

Note that it is very possible that the number of decision points, and consequently the number of different LLHs selected, that will be really executed can be, in many situations, less than the value $m d$ given as input to our approach. The selected LLH, sel, is then executed (executeLLH in Algorithm 1) considering the population size, $z$, the number of iterations/LLH, $n$, and the 
problem instance, $\mathrm{Pr}$. $\mathrm{Pop}_{h}$ is really effective after the execution of the first selected LLH and its function is related to the sharing of populations through the decision points.

Within our acceptance approach (lines from 20 through 39), the population due to the first selected LLH is always accepted, and its respective performance is positive $\left(\mathrm{Per}_{k}=\mathrm{T}\right.$ (True)). The group decision acceptance (groupDecAcc in Algorithm 1) takes place to decide whether the offspring population must be accepted. The input version of the algorithm dictates the acceptance mechanism (more details in Section 3.4). However, regardless of the version (HRISE_M, HRISE_R, HRMA), we defined a two-level (hierarchical) acceptance mechanism where in the first stage we realise whether a quality indicator improvement (onlyImproving in Algorithm 1) happens. Considering the hypervolume [35], the hypervolume improvement, $\delta\left(h_{j}\right)$, is accepted if the following condition is satisfied:

$$
\delta\left(h_{j}\right)=\frac{h_{j}-a_{|A Q|}}{a_{|A Q|}} \geq \gamma
$$

where $h_{j}$ is the hypervolume of the current decision point, $a_{|A Q|}$ is the hypervolume of the last accepted population which is stored in the sequence $A Q$, and $\gamma$ is a threshold (note that this is the same parameter we have just defined above within the nonuniform strategy). Only if this condition holds is that the group acceptance criterion, i.e. the second level, is applied. Considering this two-level acceptance mechanism (Only Improving and group decision), it is more demanding for a population to be accepted.

If a population is accepted, the current hypervolume $\left(E Q_{j}\right)$ is added to $A Q$ and the last performance of the LLH $k$ is positive $(T)$. The final population and output of our hyper-heuristics is $P o p_{f}$ while $P o p_{h}$ is a population that is considered as the initial from the second decision point onward, and whose value is adjusted before the execution of a new selected LLH.

The performance of an LLH $k$ is negatively recorded ( $\perp$, False) as well as it is penalised in accordance with the Reinforcement Learning approach (Section 3.3) in any situation in which the new set of solutions is rejected.

\subsection{The Relative Performance Per Objective Indicator}

Capacity metrics [37, such as Overall Nondominated Vector Generation (ONVG) [38, Overall Nondominated Vector Generation Ratio (ONVGR) [38, and Ratio of Nondominated solutions (RNI) [39], require that populations have nondominated solutions to work out. However, if we run a MOEA for very few iterations considering a problem instance, no solution may dominate another one and hence these indicators are of no value in these situations.

Popular convergence metrics, such as Generation Distance (GD) [40, $\epsilon$ and $\epsilon+$ indicators [1], Seven Points Average Distance (SPAD) [42], convergence-diversity metrics, such as hypervolume (s-metric) [35], Inverted Generational Distance (IGD) [43], Modified Inverted Generational Distance (IGD+) 44, and diversity metrics, such as Uniform Distribution (UD) 39, spread $(\Delta)$ 
[18] and generalised spread $\left(\Delta^{*}\right)$ [45, demand the existence of a True Pareto Front or Reference Set (reference points) which are not available for real-world problems. Even if there are practical mechanisms to overcome this issue if we think, again, about a initialisation process with very low computational demand (very few iterations) to select an LLH, these metrics may not be suitable.

Hence, we propose the Relative Performance Per Objective (RPO) indicator which does not demand a True Pareto Front, Reference Set (reference points) or nondominance of solutions, and it is suitable for a initialisation process where we run all LLHs only once and for very few iterations. Assuming all objective functions need to be minimised, RPO is defined as shown in Algorithm 2 where $F_{m \times l}$ is a $m \times l$ matrix containing the maximum values of all $m(1 \leq i \leq m)$ objective functions due to the execution of all $l$ LLHs $(1 \leq k \leq l)$.

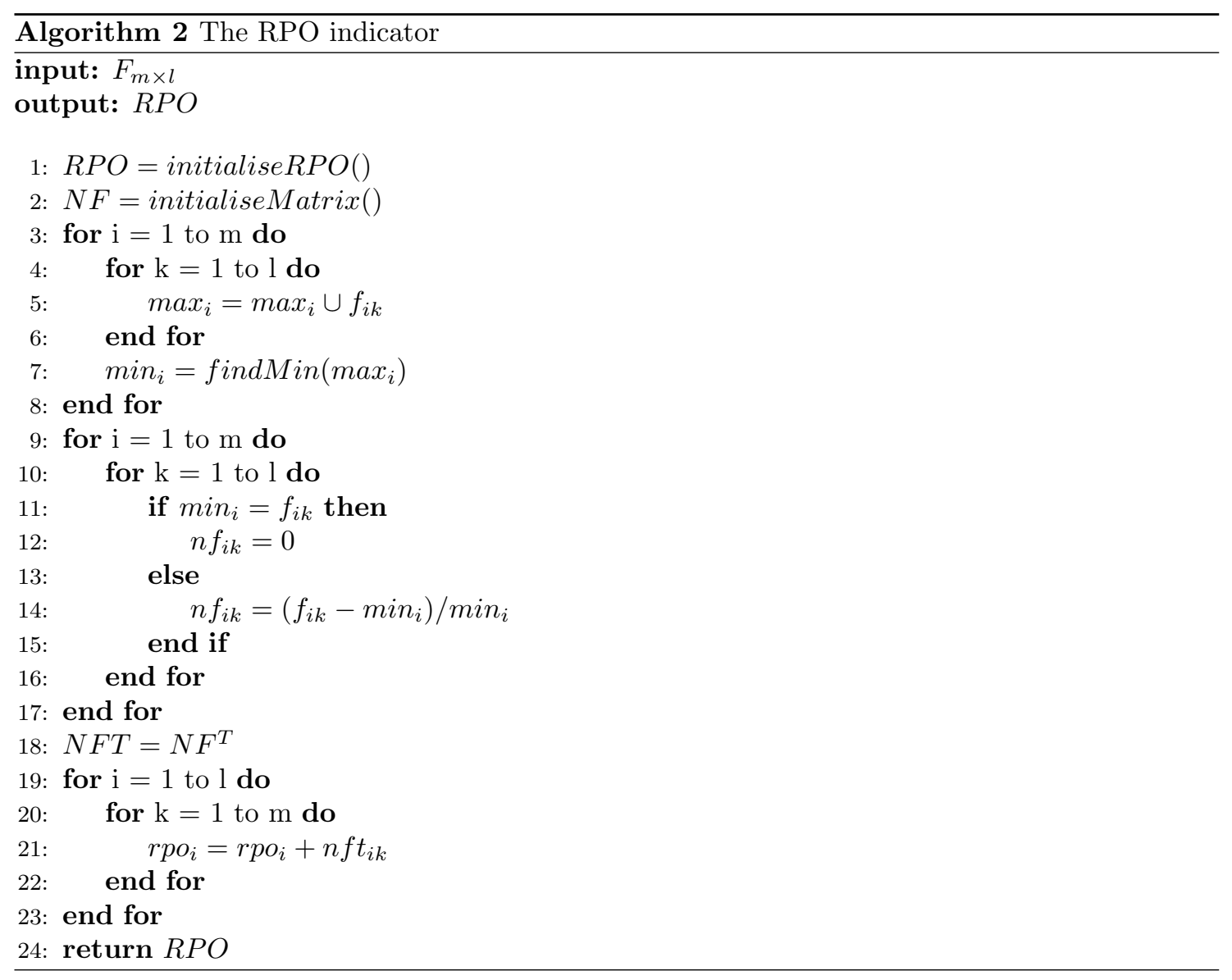

In Algorithm 2. $N F_{m \times l}$ is a matrix with the same dimensions of $F_{m \times l}$ obtained by normalising each element of $F_{m \times l}$ per objective, $N F_{l \times m}^{T}$ is its transpose, and $r p o_{i} \in R P O$. The lower the $r_{p o}$, the better the LLH. The main reasoning behind RPO is to calculate the minimum of the maximum values of the objective functions related to a population.

Let us assume that a problem instance has four objective functions $(m=4)$ and three LLHs (MOEAs) are used within our hyper-heuristics $(l=3)$. Suppose that the maximum values of 
objective functions of a population obtained by running each LLH only once and for very few iterations are as shown in matrix $F_{m \times l}$ below:

$$
F_{m \times l}=\left(\begin{array}{ccc}
1684.22 & 1681.65 & 1692.38 \\
9.08 & 8.28 & 10.65 \\
0.26 & 0.24 & 0.23 \\
8.11 & 8.15 & 7.22
\end{array}\right)
$$

Matrix $N F_{m \times l}$ is then created (lines 9 to 17 in Algorithm 2) by considering the minimum of the maximum values for each objective independently. For instance, let us consider the first objective function (row 1 of matrix $F_{m \times l}$ ). The minimum value of objective function is due to the LLH $2=1681.65$. Thus, this becomes 0 in $N F_{m \times l}$, the remaining elements of the matrix are obtained by normalisation (line 14 in Algorithm 2), and the entire $N F_{m \times l}$ is presented below:

$$
N F_{m \times l}=\left(\begin{array}{ccc}
0.001528 & 0 & 0.006381 \\
0.096618 & 0 & 0.286232 \\
0.130435 & 0.043478 & 0 \\
0.123269 & 0.128809 & 0
\end{array}\right)
$$

Matrix $N F_{l \times m}^{T}$ is the transpose of $N F_{m \times l}$ as shown below:

$$
N F_{l \times m}^{T}=\left(\begin{array}{cccc}
0.001528 & 0.096618 & 0.130435 & 0.123269 \\
0 & 0 & 0.043478 & 0.128809 \\
0.006381 & 0.286232 & 0 & 0
\end{array}\right)
$$

Each row of $N F_{l \times m}^{T}$ has the normalised values of the objective functions due to each LLH. In order to obtain the rpo of each LLH, we simply sum the elements of each row. Hence, these are the $r p o$ values for this example: $r p o_{1}=0.35185, r p o_{2}=0.172287, r p o_{3}=0.296213$. Since the smallest value is due to the LLH $2\left(\mathrm{rpo}_{2}\right)$, then this is the one chosen to be the first to be executed.

\subsection{The Balanced Exploitation/Exploration Method}

We designed additional tasks to have a balanced solution regarding exploitation $\times$ exploration as shown in Algorithm 3 . These are policies $(P 1, P 2, P 3)$ that may alter the selected LLH due to roulette wheel in the previous step. Note that some input parameters in Algorithm 3 are handled by these three policies and, as we will explain in Section 5.1 $\eta_{r}$ is a parameter that we do not need to tune because its value is chosen based on $\eta_{a}$.

Since the Reinforcement Learning mechanism (more details in Section 3.3) rewards or penalises the utility values, it is possible that, for a given problem instance, a certain LLH $k$ is selected to 275 run several times and eventually this may be the only selected LLH for this instance resulting in 


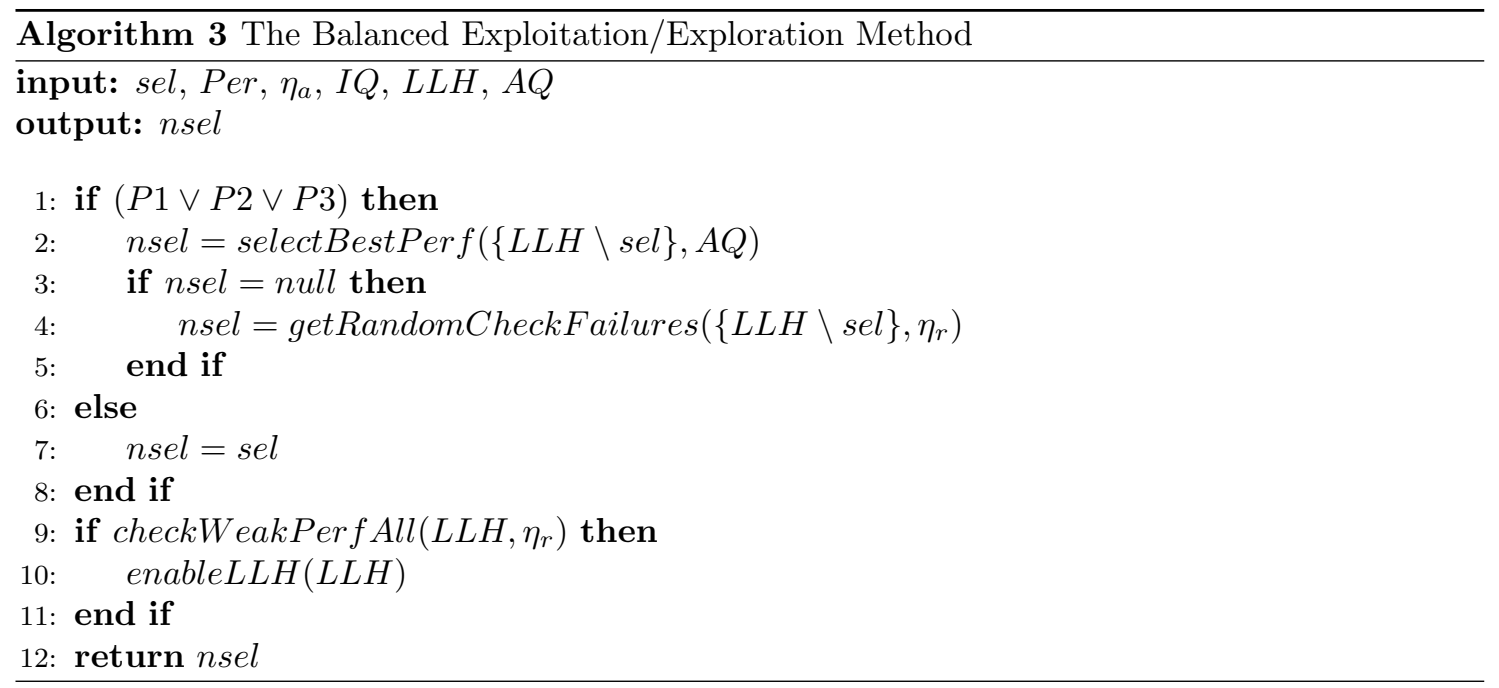

an extreme exploitation situation. This can happen because each time an LLH $k$ has its offspring population accepted, its utility value is rewarded while the others LLHs stay with the same utility value.

In order to avoid this situation, in policy $P 1$ we count the number of times (frequency) an LLH $k$ was selected to execute and verify whether the current selected LLH is above a certain limit $\left(\eta_{a}\right)$, and if this is so, another LLH must be chosen to run. An exception for this is whether the quality improvement of the last time $k$ was run is the best overall compared to the ones of the other LLHs when they were last executed (sequence $I Q$ contains such information). In this case, $k$ is still allowed to run even if its number of executions exceeds $\eta_{a}$.

Policy $P 2$ checks whether the last performance of the selected LLH was not good enough so that its population was rejected (check whether $\operatorname{Per}_{k}=\perp$ ). In policy $P 3$, we try to avoid an unnecessary exploration situation, where LLHs that have been continuously failing (i.e. populations not accepted) are ruled out if the number of failures exceed other parameter $\eta_{r}$. However, this elimination is only performed if, at the end, at least 2 LLHs are enabled for the heuristic selection process in the next decision points.

Any of these three policies being hold is enough to change the previously selected LLH, sel. A new LLH, nsel, is then chosen based on the best performance up to now (selectBestPerf in line 2 ). In other words, the decision for a certain algorithm, among the ones remaining $(\{L L H \backslash s e l\})$, is taken considering the LLH which had its population accepted in highest number of times so far. In the situation where none of the remaining LLHs is superior overall, a random LLH is chosen among them $(\{L L H \backslash s e l\})$ but still considering whether the number of failures do not exceed $\eta_{r}$ (getRandomCheckFailures in line 4).

However, the possible elimination of an LLH of the selection process may not be definitive as presented in other studies [16. Since we still continue counting the number of times the remaining 
Table 1: Some decision points to illustrate the balanced exploitation/exploration method. The $*$ means this is the outcome of the initialisation process

\begin{tabular}{c|c|c|c|c|c|c|c|c|c|c}
\hline DP & LLH-RW & $f_{k}$ & Per $_{k}$ & $I Q_{k}$ & $P 1$ & $P 2$ & $P 3$ & LLH-Bal & Pop Acc & $I Q_{k}-$ Post \\
\hline 1 & $2^{*}$ & - & - & - & - & - & - & - & YES & - \\
2 & 2 & 1 & $\mathrm{~T}$ & - & $\mathrm{F}$ & $\mathrm{F}$ & $\mathrm{F}$ & 2 & $\mathrm{NO}$ & $2 \times 10^{-5}$ \\
3 & 1 & 1 & - & - & $\mathrm{F}$ & $\mathrm{F}$ & $\mathrm{F}$ & 1 & $\mathrm{YES}$ & $8 \times 10^{-5}$ \\
4 & 1 & 2 & $\mathrm{~T}$ & $8 \times 10^{-5}$ & $\mathrm{~F}$ & $\mathrm{~F}$ & $\mathrm{~F}$ & 1 & $\mathrm{NO}$ & $1 \times 10^{-5}$ \\
5 & 1 & 3 & $\perp$ & $1 \times 10^{-5}$ & $\mathrm{~T}$ & $\mathrm{~T}$ & $\mathrm{~F}$ & 2 & $\mathrm{YES}$ & $12 \times 10^{-5}$ \\
6 & 2 & 3 & $\mathrm{~T}$ & $12 \times 10^{-5}$ & $\mathrm{~F}$ & $\mathrm{~F}$ & $\mathrm{~F}$ & 2 & $\mathrm{NO}$ & $0.5 \times 10^{-5}$ \\
7 & 3 & 1 & - & - & $\mathrm{F}$ & $\mathrm{F}$ & $\mathrm{F}$ & 3 & $\mathrm{YES}$ & $13 \times 10^{-5}$ \\
8 & 1 & 1 & $\perp$ & $1 \times 10^{-5}$ & $\mathrm{~F}$ & $\mathrm{~T}$ & $\mathrm{~F}$ & 2 & $\mathrm{NO}$ & $3 \times 10^{-5}$ \\
9 & 2 & 1 & $\perp$ & $3 \times 10^{-5}$ & $\mathrm{~F}$ & $\mathrm{~T}$ & $\mathrm{~F}$ & 3 & $\mathrm{YES}$ & $15 \times 10^{-5}$ \\
\hline
\end{tabular}

We consider $\eta_{a}=\eta_{r}=2$ for this analysis. First note that, in the first decision point, the selected LLH is not the outcome of roulette wheel but rather is the result of the initialisation process previously described and supported by the RPO quality indicator (Section 3.1). We assume that the LLH 2 is the one the initialisation process suggests as shown in the last section. performance is True $\left(\mathrm{Per}_{2}=\mathrm{T}\right.$; column $\left.\mathrm{Per}_{k}\right)$ because every population of the first decision point is always accepted. All policies are False and hence there is no change in the selected LLH and LLH 2 is indeed executed (see column $L L H-B a l$ ). But, we assume that its population is not accepted by our two-level acceptance mechanism. In decision point 3, roulette wheel selects LLH decision point, LLH 1 is again selected by roulette wheel, there is no change in the LLH, but now the population of LLH 1 is not accepted.

In decision point 5 , since roulette wheel selects again LLH 1 , we see that $f_{1}=3$ (column $f_{k}$ ) is greater than $\eta_{a}=2$. But, there is still a possibility to allow LLH 1 to run depending on the 325 quality improvement of the last time LLH 1 was run. But observe that $I Q_{1}=1 \times 10^{-5}$ (column 
$\left.I Q_{k}\right)$ while $I Q_{2}=2 \times 10^{-5}$ (column $\left.I Q_{k}-P o s t\right)$. Since the quality improvement of LLH 1 is not better than the one of LLH 2, hence another heuristic must be chosen because policy $P 1$ is indeed True. Note that policy $P 2$ is also True because of the bad last performance of LLH 1 . At this point LLH 2 had 1 population accepted (in the first decision point) and LLH 3 was never run. and Unsupervised Learning. In Reinforcement Learning, problems involve learning of what should be done (how to map situations to actions) in order to maximise a numerical reward signal [46]. They are closed-loop problems because the application of actions influences the inputs of the system in a later phase.

We use Reinforcement Learning to reward or penalise a certain LLH which is selected to run (action). Hence, if the application of an LLH $k$ results in a population that is accepted by the group decision acceptance methods (see Section 3.4), hence we reward the utility value of the LLH as:

$$
\mu^{\prime}(k)=\mu(k)+\alpha \times\left(1-\frac{j}{\lfloor 1.5 \times m d\rfloor}\right),
$$

350

where $\mu^{\prime}(k)$ and $\mu(k)$ are the next and the current utility value of the LLH $k$, respectively, $j$ is the current decision point, $m d$ is the maximum number of decision points, $\lfloor$.$\rfloor is the floor function,$ and $\alpha$ is a parameter in $[0,1]$.

If the population of a selected LLH is not accepted, it is penalised as follows:

$$
\mu^{\prime}(k)=\mu(k)-\alpha \times\left(1-\frac{j}{\lfloor 1.5 \times m d\rfloor}\right) .
$$


The utility values impact on the selection of the LLH to run in the next decision point. We only reward or penalise an LLH once per decision point within each hyper-heuristic (HRISE_M

355 a negative performance of an LLH. However, within the groupDecAcc procedure, rewardUV and penaliseUV can be called too, depending on whether an LLH must be rewarded or penalised, respectively.

\subsection{Group Decision-Making for Acceptance}

In group decision-making, several individuals participate in a process where they analyse problems, point out alternative courses of actions, and select from these alternatives one or more solutions [47. Hence, the idea is to give the responsibility to make decisions to a group of persons rather than a single individual, although this is also an option.

A previous work on selection hyper-heuristic studied ensemble move acceptance methods com-

Three move acceptance methods are embedded within HRISE/HRMA where two out of them are new ones created by varying previous solutions. Great Deluge Acceptance (GDA) always accepts improving moves and accepts moves that are worse but which are below a certain threshold which is decreased over time at a linear rate [3, 32]. It is an optimisation approach which has been demonstrating good performance in the context of selection hyper-heuristics [48].

We propose a new move acceptance method, called Qualified Late Acceptance (QLA), which is a variation of Late Acceptance (LA) [21, 22]. In LA, a comparison is made between a current generated solution and one created $s$ steps before and which is stored in a memory. The parameter $s$ is the memory length and it may influence the performance of the hyper-heuristic. Eventually, if the memory length is high, it is possible that the current generated solution is accepted not because it is too good but because the quality of the solution $s$ steps before may be too low, and hence it is easier to be beaten.

To make a comparison to more fitted populations, we store in memory ( $Q A$ in Algorithm 1) 
only the quality indicator values of the populations that were accepted in the past. Thus, in the new method QLA, we compare the quality of the current generated solution (population) to the

390 points. If the current generated solution (population) is no better than the one accepted and created $s \leq j$ steps before, hence we do not take it into account and go on.

We propose another move acceptance method called Mean Qualified Late Acceptance (MQLA). The idea is that a solution (population) is accepted if it is better than the mean value of the quality of the already accepted populations which are stored in the memory.

In HRISE_M, success is where at least two out of the three move acceptance methods agree in accepting the population. In the responsibility version, HRISE_R, only one needs to accept the population. We defined a sequence of applications of the methods in this case as: GDA, QLA, MQLA. Hence, if GDA accepts then the decision of HRISE_R is True. Otherwise, it tries between GDA, QLA, and MQLA.

Recall that we have a two-level acceptance mechanism where the group decision features (second level) are considered only if the Only Improving criterion (first level) is satisfied. The reasoning behind this strategy is to be more confident when accepting a population and avoid that weak ones to survive further in the process.

\section{Problems}

All algorithms were evaluated against seven Deb-Thiele-Laumanns-Zitzler (DTLZx, $\mathrm{x}=1 \ldots 7$ ) [49] and nine Walking Fish Group (WFGx, $x=1 \ldots 9$ ) benchmark problem instances [50]. The same parameter values for these benchmark functions were used as suggested in [49] and [50] for 410 DTLZ and WFG, respectively.

Still on the benchmark problems, we also assessed three constrained problem instances as shown in [51]: one Type-1 constrained problem instance (C1-DTLZ1) and two Type-2 constrained problem instances (C2-DTLZ2 and Convex C2-DTLZ2). In Type-1 constrained problem instances, the original True Pareto Front is still optimal, but there is an infeasible barrier in approaching it. Type-2 constrained problems introduce infeasibility to a part of the True Pareto Front and their aim is to test the ability of an algorithm to deal with discontinuities in the True Pareto Front. Moreover, we also considered the first five unconstrained ( $\mathrm{UFx}, \mathrm{x}=1 \ldots 5$ ) multi-objective problem instances from the CEC 2009 Special Session and Competition benchmark [52]. Altogether, we dealt with 24 benchmark problem instances from four problems.

The reasons for choosing the DTLZ and WFG problems and the problem instances above are because they were exactly the same as those used to evaluate HH-RILA [16. We believe that this option has allowed us to have a more adequate comparison to this recently proposed 
hyper-heuristic. Furthermore, we selected the DTLZ constrained problem instances because we had already used its unconstrained counterpart, and hence we would like to realise about the performance considering the constrained cases. Problem instances from the CEC 2009 benchmark were considered to increase even more the diversity of case studies.

We also evaluated four different real-world problems as described below. As we will explain, two of these problems are constrained. A total of 15 real-world problem instances from four problems were assessed. Altogether, we evaluated 39 problem instances from eight problems where four problems are real-world applications (unconstrained and constrained). Hence, we have $61.5 \%$ of problem instances from benchmark problems and $38.5 \%$ of problem instances from real-world applications. This relatively balanced proportion, between benchmark and real-world problems, was one of our goals. In other words, we would not like to have many more problem instances from one type than from another.

\subsection{Vehicle Crashworthiness Problem}

Structural optimisation for Vehicle Crashworthiness (VC) criteria is very relevant in the automotive industry [53. It is difficult to obtain accurate results in these types of problems due to high nonlinearities related to this context. This problem has three objective functions to be minimised: weight $\left(f_{1}\right)$, acceleration characteristics $\left(f_{2}\right)$, and toe-board intrusion $\left(f_{3}\right)$. There are five decision variables and no constraints. The objective functions are:

$$
\begin{gathered}
f_{1}(\mathbf{x})=1640.2823+2.3573285 x_{1}+2.3220035 x_{2} \\
+4.5688768 x_{3}+7.7213633 x_{4}+4.4559504 x_{5} \\
f_{2}(\mathbf{x})=6.5856+1.15 x_{1}-1.0427 x_{2}+0.9738 x_{3}+0.8364 x_{4}-0.3695 x_{1} x_{4} \\
+0.0861 x_{1} x_{5}+0.3628 x_{2} x_{4}-0.1106 x_{1}^{2}-0.3437 x_{3}^{2}+0.1764 x_{4}^{2} \\
f_{3}(\mathbf{x})=-0.0551+0.0181 x_{1}+0.1024 x_{2}+0.0421 x_{3} \\
-0.0073 x_{1} x_{2}+0.024 x_{2} x_{3}-0.0118 x_{2} x_{4}-0.0204 x_{3} x_{4} \\
-0.008 x_{3} x_{5}-0.0241 x_{2}^{2}+0.0109 x_{4}^{2}
\end{gathered}
$$

We created four problem instances related to $\mathrm{VC}$ considering the original one (three-objective) and by combining pairs of objectives: $\mathrm{VC} 1=\left\{f_{1}, f_{2}, f_{3}\right\}$; $\mathrm{VC} 2=\left\{f_{1}, f_{2}\right\}$; $\mathrm{VC} 3=\left\{f_{1}, f_{3}\right\}$; and $\mathrm{VC} 4=\left\{f_{2}, f_{3}\right\}$. 


\subsection{Water Resource Planning Problem}

Water Resource (WR) systems are crucial for a proper developed of a nation. However, issues such as inappropriate and/or degraded infrastructure, excessive withdrawals of river flows, pollution from industrial and agricultural activities are still impediments for regions around the world to have even basic drinking water and sanitation needs [54]. This problem has five objective functions that all need to be minimised: the drainage network cost $\left(f_{1}\right)$, the storage facility cost $\left(f_{2}\right)$, the treatment facility cost $\left(f_{3}\right)$, the expected flood damage cost $\left(f_{4}\right)$, and the expected economic loss due to flood $\left(f_{5}\right)$. There are 7 constraints and three decision variables [55]. The five objective functions are shown below.

$$
\begin{gathered}
f_{1}(\mathbf{x})=106780.37 \times\left(x_{2}+x_{3}\right)+61704.67 \\
f_{2}(\mathbf{x})=3000 x_{1} \\
f_{3}(\mathbf{x})=\frac{305700 \times 2289 x_{2}}{(0.06 \times 2289)^{0.65}} \\
f_{4}(\mathbf{x})=250 \times 2289 \times e^{\left(-39.75 x_{2}+9.9 x_{3}+2.74\right)} \\
f_{5}(\mathbf{x})=25 \times\left(\frac{1.39}{x_{1} x_{2}}+4940 x_{3}-80\right)
\end{gathered}
$$

We created five problem instances related to WR considering the original one (five-objective) and by combining four out of the five objectives: WR $1=\left\{f_{1}, f_{2}, f_{3}, f_{4}, f_{5}\right\}$; WR $2=\left\{f_{1}, f_{2}, f_{3}, f_{4}\right\}$; $\mathrm{WR} 3=\left\{f_{1}, f_{2}, f_{4}, f_{5}\right\} ; \mathrm{WR} 4=\left\{f_{1}, f_{3}, f_{4}, f_{5}\right\}$; and WR5 $=\left\{f_{2}, f_{3}, f_{4}, f_{5}\right\}$.

\subsection{Car Side Impact Problem}

Another problem related to the automative industry, Car Side (CS) impact deals with the optimisation of a vehicle side impact crashworthiness, but the formulation of this problem presents 10 constraints [56]. It has three objective functions that need to be minimised: the weight of car $(f 1)$, the pubic force experienced by a passenger $\left(f_{2}\right)$, and the average velocity of the $\mathrm{V}$ Pillar responsible for withstanding the impact load $\left(f_{3}\right)$. There exist 7 decision variables and the objective functions are as follows.

$$
f_{1}(\mathbf{x})=1.98+4.9 x_{1}+6.67 x_{2}+6.98 x_{3}+4.01 x_{4}+1.78 x_{5}+0.00001 x_{6}+2.73 x_{7}
$$




$$
\begin{gathered}
f_{2}(\mathbf{x})=4.72-0.5 x_{4}-0.19 x_{2} x_{3} \\
f_{3}(\mathbf{x})=0.5 \times\left(27.03-0.674 x_{1} x_{2}-0.67275 x_{2}-0.489 x_{3} x_{7}-0.843 x_{5} x_{6}\right)
\end{gathered}
$$

Three problem instances were created in addition to the original one: $\mathrm{CS} 1=\left\{f_{1}, f_{2}, f_{3}\right\}$; CS2 $=\left\{f_{1}, f_{2}\right\} ;$ and $\mathrm{CS} 3=\left\{f_{1}, f_{3}\right\}$.

\subsection{Space Applications Communication Problem}

Space Applications (SP), such as satellites, rockets, and balloons, are real-time systems. Data that are gathered by the their various computing subsystems serve not only to make decisions automatically during the operation of the systems but, naturally, they must be sent to ground stations on the Earth's surface so that controllers of such systems can perceive their health and interfere if something is wrong. Furthermore, scientists can make studies based on the observations collected by the applications.

This problem refers to real-time TeleMetry (TM) data transmission from a hard X-ray imaging telescope which is to be launched by a balloon and will operate between 40 to $42 \mathrm{~km}$ of altitude [57, 58. The On-Board Data Handling Subsystem (OBDH) is responsible for acquiring, formatting, and transmitting to the Ground Station (GS) all TM data generated by several subsystems (X-ray Camera (XRC), Attitude Control Subsystem (ACS)) of the space segment. The OBDH continuously stores on-board and sends to the GS all TM data it obtains. The main goal is trying to minimise the total time (associated with transmission and propagation delays) of these data that are stored on-board and, at the same time, are visualised on the GS in real-time.

There are three objective functions: minimise the total time related to the delivery of scientific data to the GS $\left(f_{1}\right)$, minimise the total time related to the delivery of housekeeping data (OBDH + ACS) to the GS $\left(f_{2}\right)$, and maximise the total amount of data transmitted to the GS $\left(f_{3}\right)$. This problem has no constraints while it has four decision variables: amount of scientific data TM packet $\left(x_{1}\right)$; amount of housekeeping data TM packet from the OBDH $\left(x_{2}\right)$; amount of housekeeping data

${ }_{485}$ TM packet from the ACS $\left(x_{3}\right)$; and amount of event packets generated by the XRC $\left(x_{4}\right)$. The objective functions are described in the sequence where $f_{3}$ is formulated as a minimisation problem too.

$$
\begin{gathered}
f_{1}(\mathbf{x})=x_{1} \times\left(x_{4}+\frac{48 x_{4}+96}{115200}+\frac{48 x_{4}+272}{500000}\right)+0.300133 \\
f_{2}(\mathbf{x})=\frac{115264 x_{2}+115328 x_{3}}{115200}+0.300133
\end{gathered}
$$




$$
f_{3}(\mathbf{x})=300-\left(x_{1}+x_{2}+x_{3}\right)
$$

Three problem instances were created: the original instance, $\mathrm{SP} 1=\left\{f_{1}, f_{2}, f_{3}\right\} ; \mathrm{SP} 2=\left\{f_{1}, f_{3}\right\}$; and SP3 $=\left\{f_{2}, f_{3}\right\}$.

490

\section{Experimental Results}

Our hyper-heuristics were implemented based on two frameworks: version 5.6 of jMetal [59. and jMetalHyperHeuristicHelper [60]. As for the experimental evaluation, we considered as LLHs three MOEAs: NSGA-II [18], IBEA [19], and SPEA2 [20]. We chose these MOEAs due to their popularity and also because several other selection hyper-heuristics [13, 15, 16] have made use of them, and so this is more suitable for the analysis. Selection of individuals was binary tournament, we used Simulated Binary Crossover (SBX) 61] with probability 0.9 and distribution index 20, and Polynomial mutation with probability $1 / n$ ( $n=$ number of parameters) and distribution index 20 . We compared our approaches to three other hyper-heuristics (HH-CF, HH-ALL, and HH-RILA), and the three MOEAs run in isolation.

We executed all approaches for each problem instance for 100,000 evaluations, population size $(z)$ was 100, and trials were 30. Hence, for our hyper-heuristics, HH-CF, HH-ALL, NSGAII, IBEA, SPEA2, the maximum number of iterations ( $m i$ ) was 1,000. Within our nonuniform strategy, the first selected LLHs run for 250 iterations and in the later decision points they run for 100 iterations (but recall that even later it is possible a huge number of iterations/LLH, i.e. 250). 16. The maximum number of decision points $(m d)$ for our hyper-heuristics is 7 , and in HH-CF it is 25 as previously mentioned [13] as well as this is the case for HH-ALL. Table 2 summarises the values of the main parameters used.

The choice of values for the common parameters was driven by other studies where some works use 50,000, 75,000, 100,000 evaluations [16, depending on the problem instance, and others use 100,000 evaluations 62]. We decided to use a fixed default value for all instances equals to 100,000 evaluations to accomplish a more uniform comparison. This choice is not related to any previous analysis of convergence of the algorithms. As well as a population of 100 solutions and 30 trials are quite common values in several other studies in the literature.

515

The tuning of parameters of our hyper-heuristics are presented in Section 5.1 while the meaning of the parameters of HH-RILA [16] and HH-CF [13, shown in Table 2, are described in their respective papers. Moreover, $\alpha$ in our hyper-heuristics has a different meaning of $\alpha$ in HH-CF. All experiments were performed on an Intel Xeon $3.5 \mathrm{GHz}$ processor with $32 \mathrm{~GB}$ of RAM memory and Windows 10 Operating System. All populations (VAR files) of all algorithms, the respective 
Table 2: Experimental evaluation: values of parameters

\begin{tabular}{|c|c|}
\hline \multicolumn{2}{|c|}{ Common Parameters } \\
\hline Number of Evaluations & 100,000 \\
\hline Population Size & 100 \\
\hline Trials & 30 \\
\hline \multicolumn{2}{|c|}{ Specific Parameters } \\
\hline \multicolumn{2}{|c|}{ HRISE_M, HRISE_R, HRMA } \\
\hline Iterations/LLH & 250 or 100 \\
\hline Maximum Iterations & 1,000 \\
\hline Maximum Decision Points & 7 \\
\hline$\alpha$ & 1.0 (HRISE_M) / 0.1 (HRISE_R) \\
\hline$\eta_{a}$ & 2 (HRISE_M) / 3 (HRISE_R) \\
\hline$\eta_{r}$ & 2 (HRISE_M) / 1 (HRISE_R) \\
\hline$\gamma$ & 0.000075 \\
\hline \multicolumn{2}{|c|}{ HH-RILA } \\
\hline Iterations/LLH & 10 \\
\hline$\tau$ & 0.9 \\
\hline$m$ & 3.0 \\
\hline$K$ & 3 \\
\hline$\Delta_{v}$ & 0.0075 \\
\hline \multicolumn{2}{|c|}{$\mathrm{HH}-\mathrm{CF}$} \\
\hline Maximum Iterations & 1,000 \\
\hline Maximum Decision Points & 25 \\
\hline$\alpha$ & 100 \\
\hline \multicolumn{2}{|c|}{ HH-ALL } \\
\hline Maximum Iterations & 1,000 \\
\hline Maximum Decision Points & 25 \\
\hline \multicolumn{2}{|c|}{ NSGA-II, IBEA, SPEA2 } \\
\hline Maximum Iterations & 1,000 \\
\hline Maximum Decision Points & 1 \\
\hline
\end{tabular}

520 Pareto Fronts (.pf files) are stored in 63.

\subsection{Parameter Tuning}

There are two main parameters to tune within our approaches: $\alpha$ and $\eta_{a}$. The $\eta_{r}$ parameter was selected based on $\eta_{a}$ :

$$
\eta_{r}=\left\{\begin{aligned}
\eta_{a}, & \text { if } \quad \eta_{a}=2 \text { (more flexible) } \\
\eta_{a}-2, & \text { if } \quad \eta_{a}>2 \text { (less flexible) }
\end{aligned}\right.
$$

When $\eta_{r}=\eta_{a}$, we say that there is a greater flexibility regarding the LLHs which perform worse, while $\eta_{r}=\eta_{a}-2$ means less flexibility because we eliminate an LLH with less number of failures (populations not accepted). Other parameters were selected based on previous studies. For instance, $\gamma=0.0075 / 100=0.000075$ since 0.0075 was the value suggested in the HH-RILA approach [16]. But in HH-RILA, the comparison is made to the hypervolume of the last population 
530 (regardless whether it was accepted or not) while we compare to the last accepted population. Thus, we gave a lower margin to avoid being too rigid to consider a population fitted.

The parameters could take the following values: $\alpha=\{0.1,0.4,0.6,1.0\}, \eta_{a}=\{2,3,4\}$. With respect to HRISE_M and HRISE_R, we ran all problem instances from two theoretical benchmarks (DTLZ, WFG) and all problem instances from VC (only) for 30 trials, for every combination ${ }_{535}\left\langle\alpha, \eta_{a}\right\rangle$.

We took into account two levels of normalisation of the hypervolume indicator. The frontnormalised hypervolume, $h$, is obtained via the normalisation of the "raw" hypervolume based on the minimum and maximum values of the objective functions of a problem instance. From this point onward, we will denote it simply as hypervolume, $h$, and as higher its value, the better.

As for the cross-domain analysis, where we aim at realising about the generalisation features of hyper-heuristics, we used the normalised (front-normalised) hypervolume, $h_{N}$, as defined below [16]:

$$
h_{N}=\frac{h_{(\forall a, p)}^{\max }-\overline{h_{\left(a_{i}, p\right)}}}{h_{(\forall a, p)}^{\max }-h_{(\forall a, p)}^{\min }}
$$

where $h_{(\forall a, p)}^{\max }$ and $h_{(\forall a, p)}^{\min }$ are the maximum and minimum values, respectively, of the hypervolume,

$h$, due to all algorithms $a$ for a problem instance $p$, and $\overline{h_{\left(a_{i}, p\right)}}$ is the average value of the hyper-

volume due to algorithm $a_{i}$ for $p$. From this point onward, we will denote it simply as normalised hypervolume, $h_{N}$. However, note that the formulation of $h_{N}$ is like a maximisation problem (maximise hypervolume) is turned into a minimisation problem. Hence, the lower the value of $h_{N}$, the better.

In order to decide the values of parameters for our approaches, we used $h_{N}$. Tuning of HRISE_M produced as a result: $\left\{\alpha=1.0, \quad \eta_{a}=2\right\}$. The choice for HRISE_R was: $\left\{\alpha=0.1, \quad \eta_{a}=3\right\}$.

\subsection{Cross-Domain Performance Analysis}

One of the main claims of hyper-heuristics supporters is generalisation, i.e. their ability to get better results across several different problems rather than a single problem domain. Hence, the cross-domain performance analysis is a very suitable evaluation tool in this context. Tables 3 and 4 present the results of the cross-domain performance analysis using as metric the normalised hypervolume, $h_{N}$, for the benchmark functions, where in Table 3 we show the results for problems DTLZ, WFG, Constrained DTLZ, and in Table 4 we show the results for the UF problem. In Table 5, we present the results for the real-world and for all (benchmark + real) problems.

In Table 3 and also in some figures later, DTx, WFx identify the DTLZ and WFG problem instance, respectively, while C1DT1 is C1-DTLZ1, C2DT2 is C2-DTLZ2, and CONVC2DT2 is the Convex C2-DTLZ2 problem instances. Moreover, $\overline{h_{N}^{D T}}, \overline{h_{N}^{W F}}$, and $\overline{h_{N}^{C D T}}$ means the average values of $h_{N}$ for DTLZ, WFG, and Constrained DTLZ, respectively. In Table 4. UFx identifies the CEC 
2009 unconstrained problem instance and $\overline{h_{N}^{U F}}$ is the average of such instances. Furthermore, in Table $4 . \overline{h_{N}^{B E N}}$ is the average value considering all 24 benchmark problem instances.

In Table 5 we see the identification of each real-world problem instance, the average value of the problem instances, $\overline{h_{N}^{y}}$, the mean of all 15 real-world problem instances, $\overline{h_{N}^{R E A}}$, and finally the average value of all 39 (benchmark + real) problem instances, $\overline{h_{N}^{A P R}}$. In grey background we show the top performance approaches where in bold it is the best algorithm while in italics it is the second best.

Note that the differences are small but this is probably a consequence of the double normalisation approach we took regarding the calculation of the hypervolume. As for the DTLZ problem, we see that HRISE_R was the best overall followed by HH-CF. The good performance of HH-CF, as being even superior to SPEA2, contradicts previous results [16]. One possible explanation for this relies on the fact that $\mathrm{HH}-\mathrm{CF}$, in many problem instances including some DTLZ ones, selected SPEA2 as the LLH to run and this may be boosted its results regarding all DTLZ problem instances. As for the WFG problem, we have observed similar results as in previous studies where IBEA presented the best performance followed by HH-RILA. Regarding the Constrained DTLZ problem, HRISE_R again produced the top performance while HRMA ranked the second. Regarding UF, NSGA-II achieved the top rank and HH-RILA was the second best approach. Across all benchmark problem instances, our hyper-heuristics performed the best where HRISE_R was the top algorithm followed by HRISE_M.

Considering the real-world problems, in order to obtain the reference points for hypervolume calculation, we created the so called True Known Pareto Front where, for each problem instance, we joined all final populations of all algorithms after the 30 trials, obtained the nondominated solutions, and removed the repeated ones. Results for the VC problem are in line with previous studies [16] where NSGA-II got the best performance followed by HH-RILA. In two problems, WR and SP, HRISE_M and HRISE_R presented the best results (HRISE_M was the best in both problems). For all real-world problem instances, HH-RILA was the best being slightly superior than HRISE_M. However, considering all 39 (benchmark + real) problem instances $\left(\overline{h_{N}^{A P R}}\right)$, HRISE_R was the best followed by HRISE_M, and HRMA.

In Table 6, we present a statistical evaluation considering the 39 problem instances altogether but we used the hypervolume, $h$, at this time where the higher the value, the better. We applied a two-tailed permutation test (conditional inference procedure) 64 for multi-group comparison with significance level equal to 0.05. In Table 6. ">" means the leftmost algorithm was significantly better than the rightmost one, " $<$ " means the leftmost algorithm was significantly worse, " $\sim$ " means no significant difference, and $\mathrm{E}$ in the p-values $(p)$ represents the scientific notation (standard form).

In accordance with the statistical outcomes, the top three approaches ranked based on the cross- 


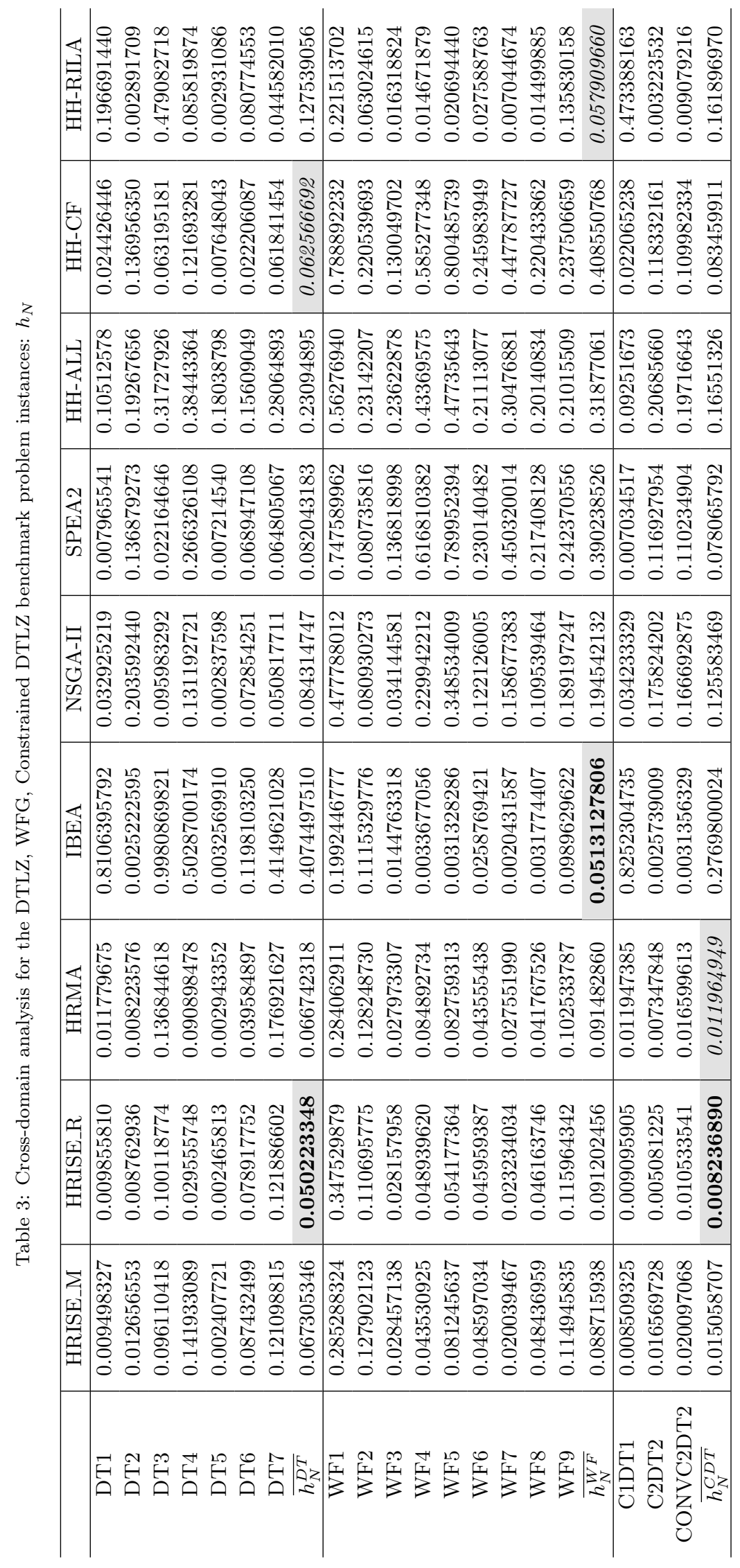




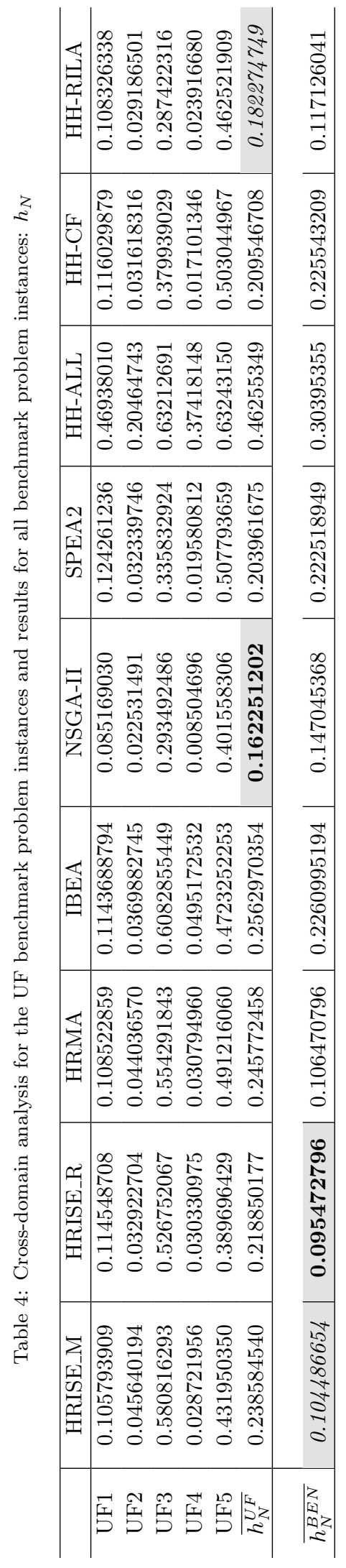




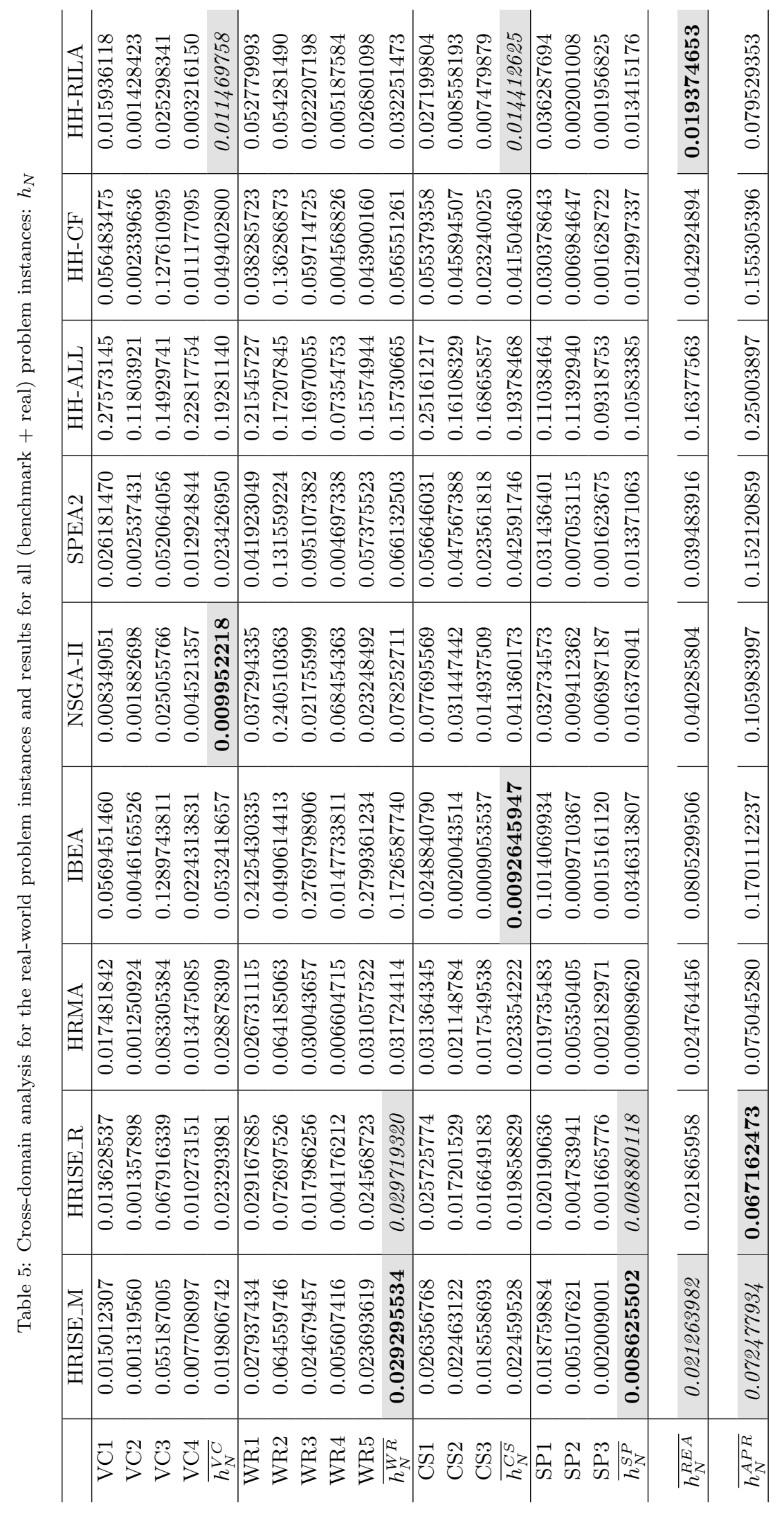


Table 6: Statistical comparison considering all (benchmark + real) problem instances: $h$. Caption: Comp $=$ Comparison; Res $=$ Result; $p=\mathrm{p}$-value

\begin{tabular}{c|c|c}
\hline Comp & Res & $p$ \\
\hline HRISE_M $\times$ HRISE_R & $\sim$ & $8.903 \mathrm{E}-01$ \\
HRISE_M $\times$ HRMA & $\sim$ & $9.180 \mathrm{E}-01$ \\
HRISE_M $\times$ IBEA & $>$ & $1.105 \mathrm{E}-09$ \\
HRISE_M $\times$ NSGA-II & $\sim$ & $2.937 \mathrm{E}-01$ \\
HRISE_M $\times$ SPEA2 & $>$ & $9.811 \mathrm{E}-03$ \\
HRISE_M $\times$ HH-ALL & $>$ & $3.996 \mathrm{E}-14$ \\
HRISE_M $\times$ HH-CF & $>$ & $2.812 \mathrm{E}-03$ \\
HRISE_M $\times$ HH-RILA & $\sim$ & $2.772 \mathrm{E}-01$ \\
HRISE_R $\times$ HRMA & $\sim$ & $8.297 \mathrm{E}-01$ \\
HRISE_R $\times$ IBEA & $>$ & $3.499 \mathrm{E}-10$ \\
HRISE_R $\times$ NSGA-II & $\sim$ & $2.200 \mathrm{E}-01$ \\
HRISE_R $\times$ SPEA2 & $>$ & $4.948 \mathrm{E}-03$ \\
HRISE_R $\times$ HH-ALL & $>$ & $7.992 \mathrm{E}-15$ \\
HRISE_R $\times$ HH-CF & $>$ & $1.264 \mathrm{E}-03$ \\
HRISE_R $\times$ HH-RILA & $\sim$ & $2.144 \mathrm{E}-01$ \\
HRMA $\times$ IBEA & $>$ & $1.777 \mathrm{E}-09$ \\
HRMA $\times$ NSGA-II & $\sim$ & $3.209 \mathrm{E}-01$ \\
HRMA $\times$ SPEA2 & $>$ & $1.289 \mathrm{E}-02$ \\
HRMA $\times$ HH-ALL & $>$ & $6.128 \mathrm{E}-14$ \\
HRMA $\times$ HH-CF & $>$ & $3.846 \mathrm{E}-03$ \\
HRMA $\times$ HH-RILA & $\sim$ & $2.958 \mathrm{E}-01$ \\
IBEA $\times$ NSGA-II & $<$ & $2.819 \mathrm{E}-07$ \\
IBEA $\times$ SPEA2 & $<$ & $1.955 \mathrm{E}-04$ \\
IBEA $\times$ HH-ALL & $\sim$ & $2.937 \mathrm{E}-01$ \\
IBEA $\times$ HH-CF & $<$ & $5.678 \mathrm{E}-04$ \\
IBEA $\times$ HH-RILA & $<$ & $1.196 \mathrm{E}-06$ \\
NSGA-II $\times$ SPEA2 & $\sim$ & $1.637 \mathrm{E}-01$ \\
NSGA-II $\times$ HH-ALL & $>$ & $3.685 \mathrm{E}-11$ \\
NSGA-II $\times$ HH-CF & $\sim$ & $7.056 \mathrm{E}-02$ \\
NSGA-II $\times$ HH-RILA & $\sim$ & $9.180 \mathrm{E}-01$ \\
SPEA2 $\times$ HH-ALL & $>$ & $1.605 \mathrm{E}-07$ \\
SPEA2 $\times$ HH-CF & $\sim$ & $7.826 \mathrm{E}-01$ \\
SPEA2 $\times$ HH-RILA & $\sim$ & $2.200 \mathrm{E}-01$ \\
HH-ALL $\times$ HH-CF & $<$ & $5.645 \mathrm{E}-07$ \\
HH-ALL $\times$ HH-RILA & $<$ & $3.520 \mathrm{E}-10$ \\
HH-CF $\times$ HH-RILA & $\sim$ & $1.118 \mathrm{E}-01$ \\
\hline & &
\end{tabular}

domain analysis, i.e. our three hyper-heuristics, were not better than HH-RILA and NSGA-II.

However, HRISE_R, HRISE_M, and HRMA were superior than all remaining algorithms, including SPEA2 and HH-CF, while there is a tie if we make a pairwise comparison between HH-RILA, NSGA-II, SPEA2, and HH-CF. In other words, between these last four algorithms in a pairwise manner (i.e. NSGA-II $\times$ SPEA2, NSGA-II $\times$ HH-CF, NSGA-II $\times$ HH-RILA, SPEA2 $\times$ HH-CF, SPEA2 $\times$ HH-RILA, HH-CF $\times$ HH-RILA), there is no statistical difference considering $h$ as a metric. Thus, from the statistical point of view, we may not claim that our hyper-heuristics were truly better than HH-RILA and NSGA-II, but we might say that they are at least slightly better 
because of the remarks we have just presented above. We also compared all algorithms via the normalised $\epsilon$ indicator $\left(\epsilon_{N}\right)$ which shares the main reasoning behind the normalised hypervolume. However, $\epsilon_{N}$ is calculated in the traditional manner in the context of minimisation problems since as lower $\epsilon$, and also $\epsilon_{N}$, the better. In Table 7, we show a summarised version of the results presenting only the average values $\left(\epsilon_{N}^{y}\right)$. As in the case of hypervolume, for the real problems, we used the True Known Pareto Front obtained along the execution of all algorithms.

Again we see the good performance of HH-CF for the DTLZ problem where it obtained the top place followed by HRISE_R. With respect to WFG, we have the same pattern as in the case of $h_{N}$ while for the Constrained DTLZ problem we note a swap between the top contenders: HRMA was the best followed by HRISE_R. As for the UF problem we notice the same classification as in the hypervolume case, and overall, for the benchmark problem instances, HH-RILA is slightly better than HRISE_R. Considering the real-world problems, we might say that our approaches were even better than in the previous analysis, where in three problems (WR, CS, and SP) they were the two best solutions, as well as HRISE_M presented the best results for all real-world problems followed by HRISE_R. Considering all 39 (benchmark + real) problem instances, HRISE_R showed the best performance followed by HH-RILA, HRISE_M, and HRMA.

The conclusion of all these results is that our hyper-heuristics presented the best results when comparing to HH-CF, HH-ALL, and the MOEAs run in isolation. Regarding the comparison to the recently proposed HH-RILA, even if we saw no statistical significance when evaluating the hypervolume, $h$, we may say that our hyper-heuristics were at least slightly better than HH-RILA taking into account the pairwise comparison between HH-RILA and some other approaches as we have previously pointed out. Overall, taking into account both quality indicators, HRISE_R was the best of all algorithms based on the results of the cross-domain evaluations.

However, we should mention that we still need to perform more experiments considering other benchmark and real-world problems to definitely conclude on the best performance of the approaches. In other words, more problems are required to conclude about generalisation. This is known as a threat to external validity related to the population in the context of controlled experiments [65, 66.

\subsection{Evaluation of the Heuristic Selection Mechanisms: Utilisation Rate}

In this section, we aim at evaluating the heuristic selection mechanisms implemented within our three hyper-heuristics and HH-RILA, the most recent and which got the closest performance in comparison with our proposals. We use the utilisation rate as our main metric over all 30 trials. Figures 3 and 4 present the results regarding the benchmark problems DTLZ and WFG, respectively. At first, it seems that HH-RILA eventually possesses more "intelligence" than our solutions since we clearly see that it favours much more some MOEAs in certain problem instances 


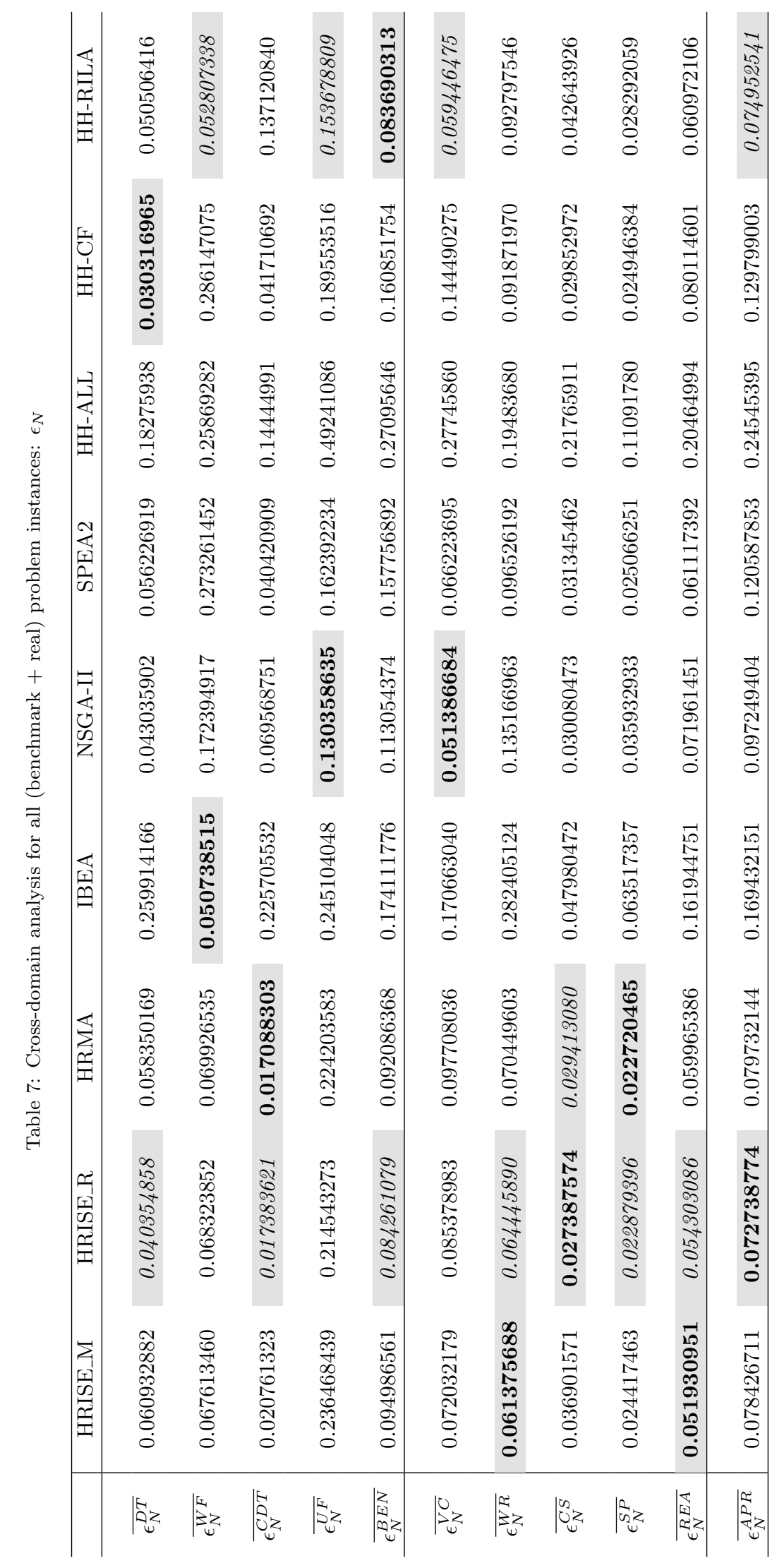


which may be a good approach. But, HH-RILA has within its initialisation process a mechanism that may rule out an LLH, depending on its performance, for all the remaining iterations. As we will mention, sometimes this is interesting and in other situations it is not.

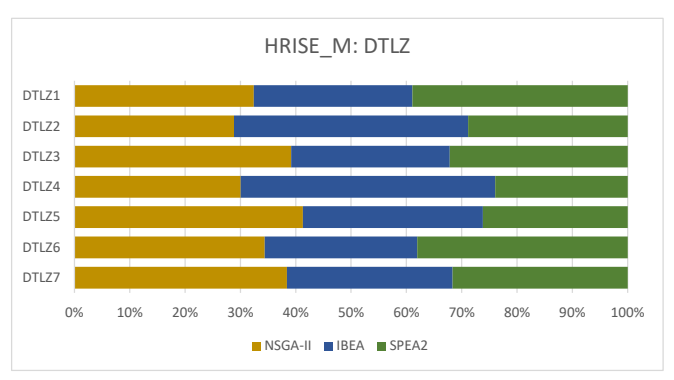

(a) Utilisation Rate: HRISE_M

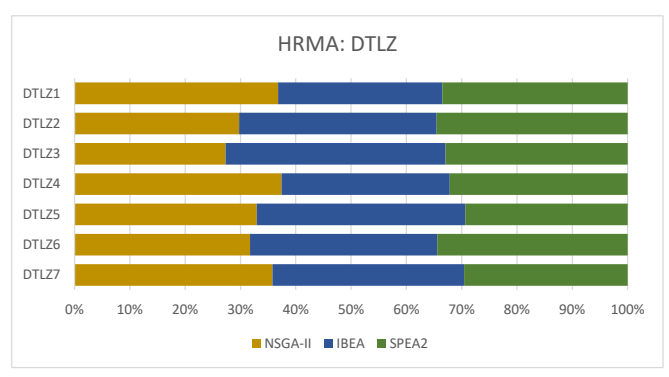

(c) Utilisation Rate: HRMA

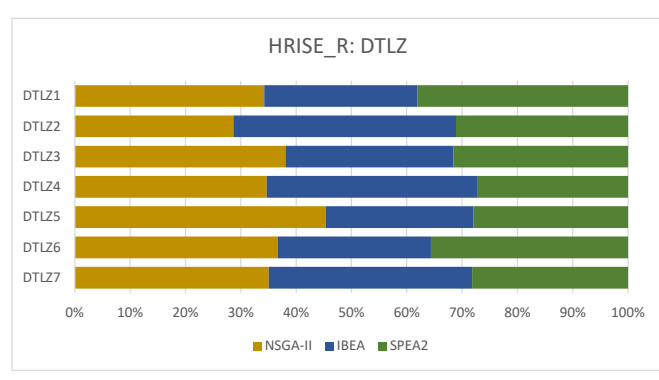

(b) Utilisation Rate: HRISE_R

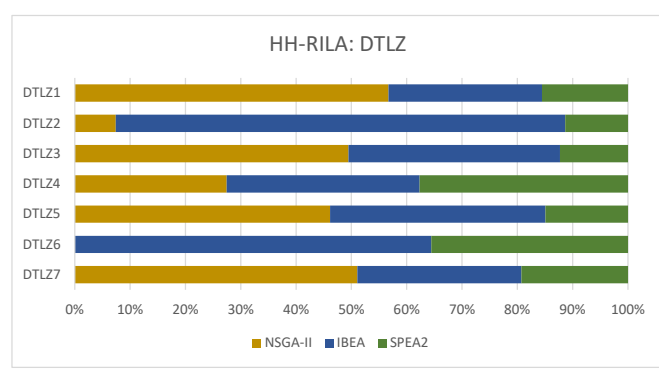

(d) Utilisation Rate: HH-RILA

Figure 3: Utilisation Rate: DTLZ problem instances

Let us consider the DTLZ6 problem instance. HH-RILA completely eliminated NSGA-II and all the iterations were split between IBEA and SPEA2. But, in Figure 5a, we see that the mean hypervolume $(h)$ of NSGA-II over 30 trials is the second best if we compare only the three MOEAs in isolation. It is better than IBEA. Hence, the radical measure to get rid off an LLH $k$ at the beginning was not a suitable measure in this case. As we have previously mentioned, in HRISE_R and HRISE_M we can eliminate an LLH $k$ but such action may be temporary because there is a possibility it comes back again, depending on the performance of the remaining MOEAs. This explains a more uniform utilisation rate within our hyper-heuristics.

As for WFG, HH-RILA was even more radical excluding NSGA-II from WFG3 up WFG8 problem instances and selecting many more times IBEA. This was a good decision since the very good outcomes produced by IBEA when applied to solve the WFG problem instances as shown in Section 5.2 However, note again that the mean hypervolume of NSGA-II was better than SPEA2 


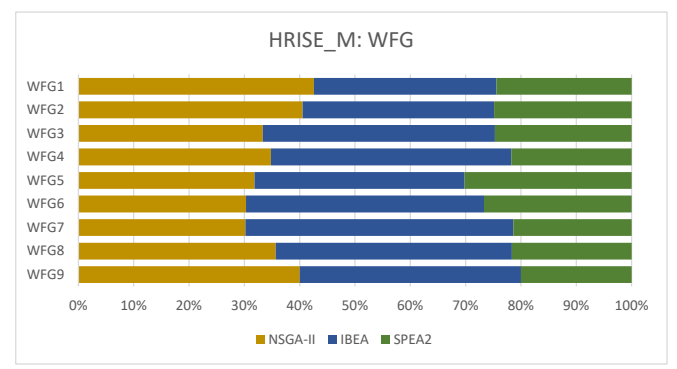

(a) Utilisation Rate: HRISE_M

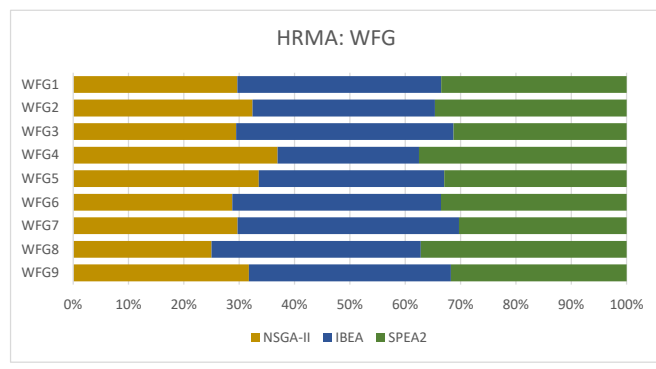

(c) Utilisation Rate: HRMA

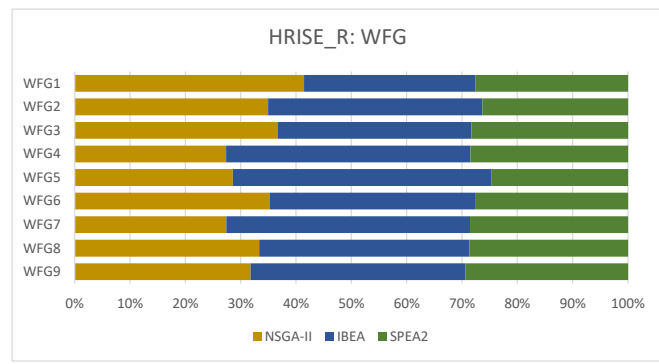

(b) Utilisation Rate: HRISE_R

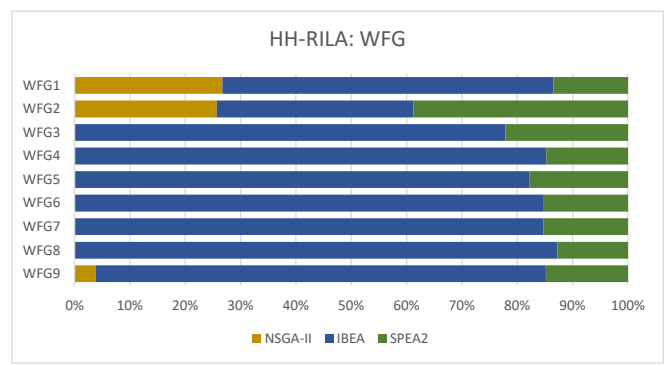

(d) Utilisation Rate: HH-RILA

Figure 4: Utilisation Rate: WFG problem instances

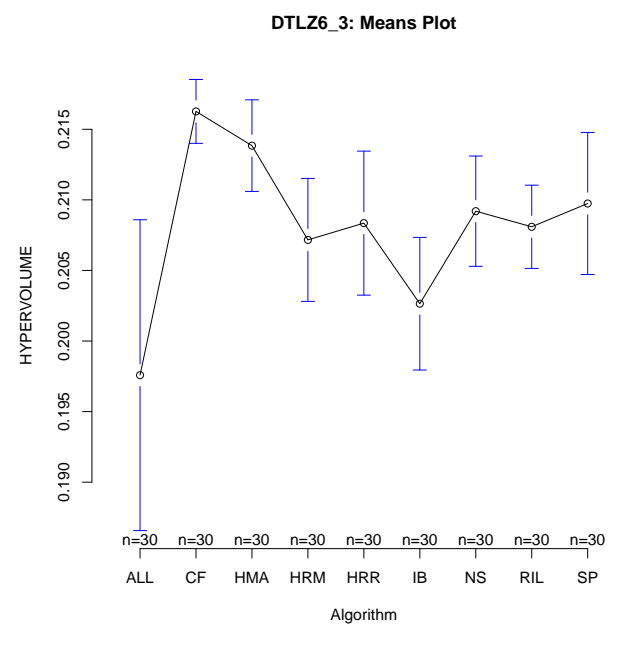

(a) DTLZ6

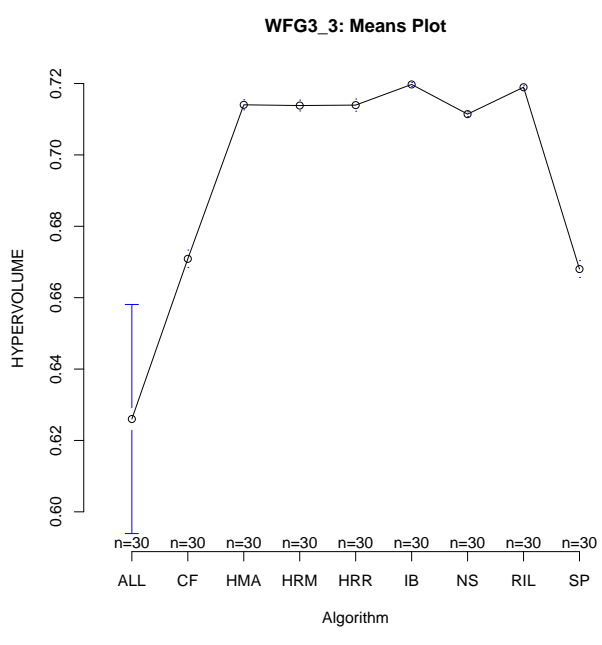

(b) WFG3

Figure 5: Means plots of the hypervolume $(h)$ over 30 trials: DTLZ6 and WFG3. The number after "" in the problem instance nomenclature is the number of objective functions, and the circle is the mean value. Caption: ALL $=$ HH-ALL; CF = HH-CF; HMA = HRMA; HRM = HRISE_M; HRR = HRISE_R; IB = IBEA; NS = NSGA-II, RIL $=$ HH-RILA; SP $=$ SPEA2 
for the WFG3 problem instance (Figure 5b).

In Figures 6 and 7 we show the utilisation rates for two real-world problems, respectively, SP and WR. As in the case of the benchmark problems, HH-RILA presented a very nonuniform LLH selection behaviour even if now only in WR5 an LLH (IBEA) was excluded definitely. As earlier, this can be suitable or not. If we consider SP2, HH-RILA selected proportionally many more times IBEA even if the mean hypervolume between the three MOEAs are very close (see Figure 8a. . On the other hand, in WR1, HH-RILA selected a few times IBEA and it seems suitable since IBEA presented the smallest of all average hypervolume values for such a problem instance (see

Figure 8b).

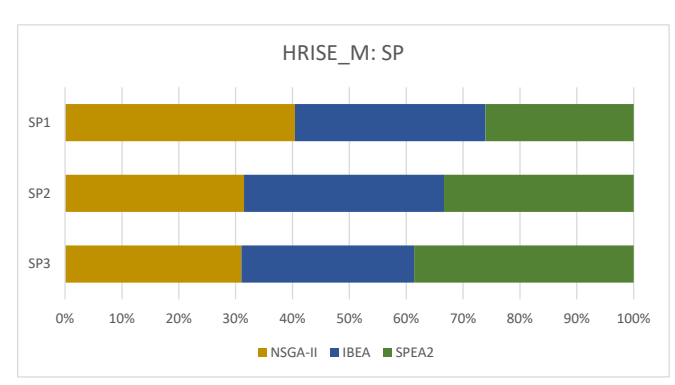

(a) Utilisation Rate: HRISE_M

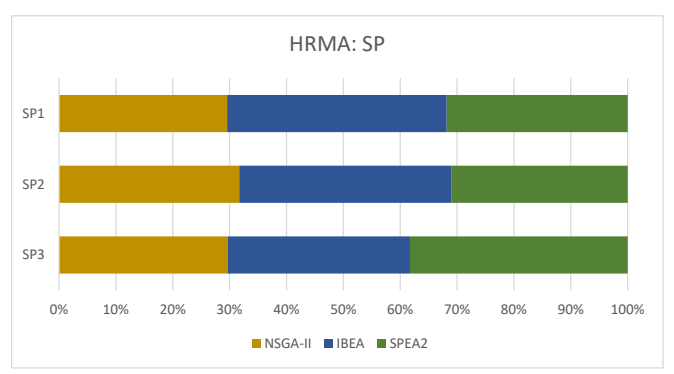

(c) Utilisation Rate: HRMA

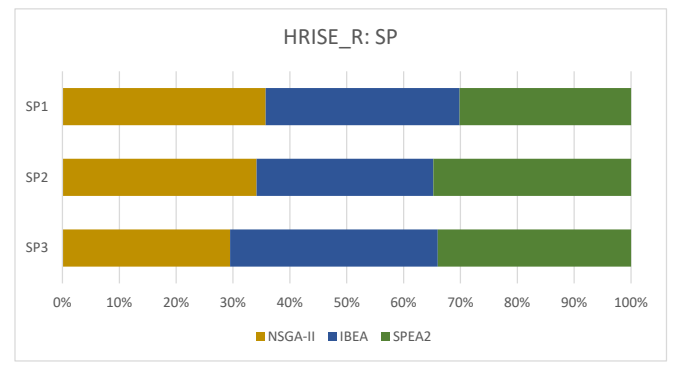

(b) Utilisation Rate: HRISE_R

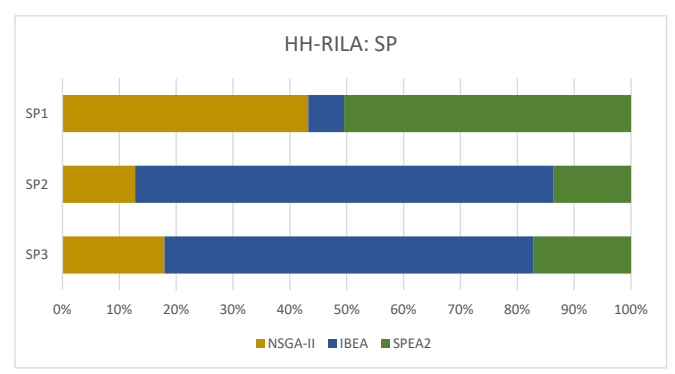

(d) Utilisation Rate: HH-RILA

Figure 6: Utilisation Rate: SP problem instances

It is not totally clear whether eliminating an LLH $k$ and avoiding it to be executed at all, as HH-RILA does, is overall a worse or better solution compared to a more permissive approach where other chances are given to an LLH that was previously ruled out, as our hyper-heuristics accomplish. This is a point that remains an open question within the context of hyper-heuristics. 


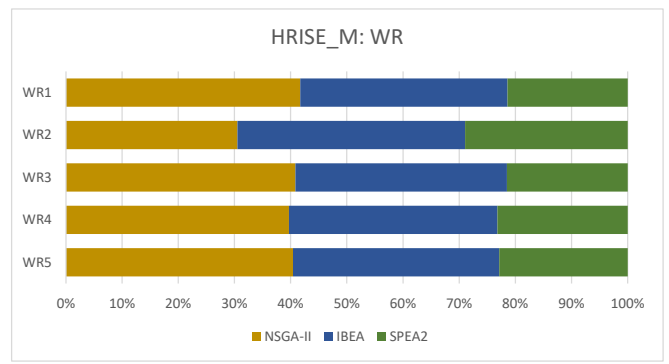

(a) Utilisation Rate: HRISE_M

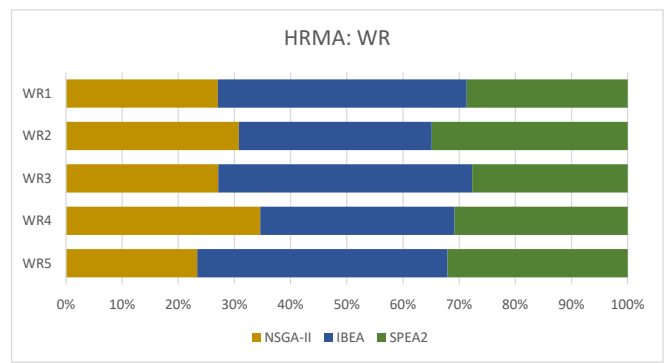

(c) Utilisation Rate: HRMA

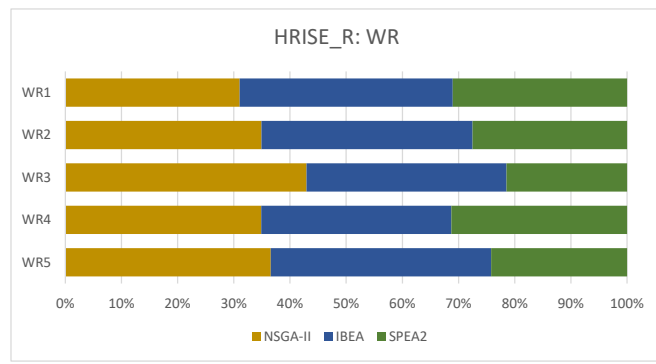

(b) Utilisation Rate: HRISE_R

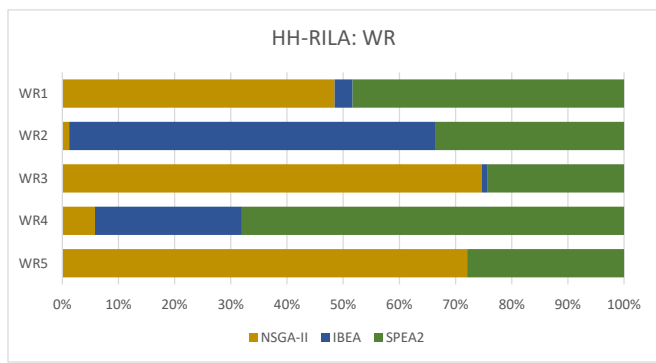

(d) Utilisation Rate: HH-RILA

Figure 7: Utilisation Rate: WR problem instances

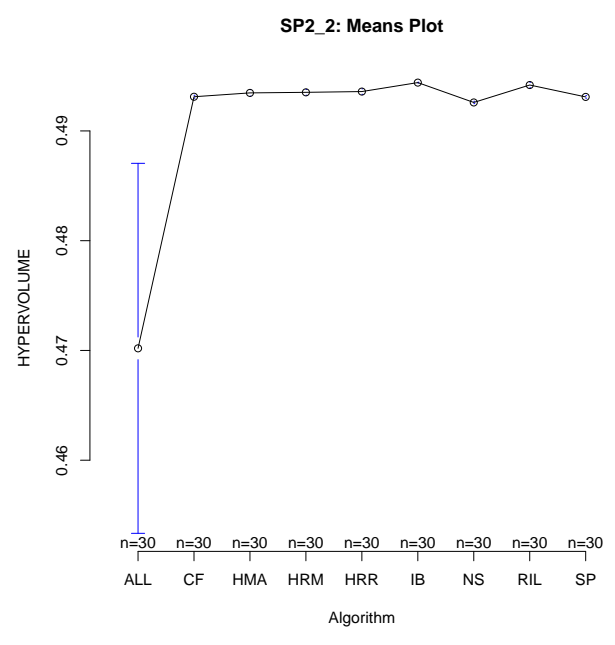

(a) SP2

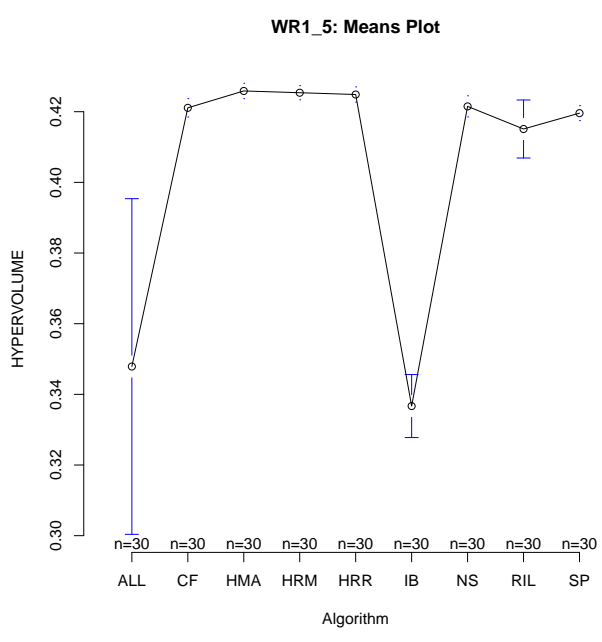

(b) WR1

Figure 8: Means plots of the hypervolume $(h)$ over 30 trials: SP2 and WR1. The number after "." in the problem instance nomenclature is the number of objective functions, and the circle is the mean value. Caption: ALL = HH-ALL; CF = HH-CF; HMA = HRMA; HRM = HRISE_M; HRR = HRISE_R; IB = IBEA; NS = NSGA-II, RIL $=$ HH-RILA $;$ SP $=$ SPEA2 
We now show some Solution Front: $3^{3}$ where our hyper-heuristics had better and worse performances compared to HH-RILA, considering the benchmark and real-world problem instances. We also show the True Pareto Front or True Known Pareto Front as references. Regarding the benchmark problem instances, DTLZ3 was one where HRISE_R and HRISE_M got better results (Figure 9). Note that HH-RILA struggled here where many points in the objective space present very low objective values given the sensation that we have only few points (Figure 9d). At first, we observed this behaviour in our hyper-heuristics too, but the nonuniform iterations strategy was crucial to overcome this issue. In Figure 10, we see a better performance of HH-RILA particularly when compared to HRISE_R for the WFG1 problem instance.

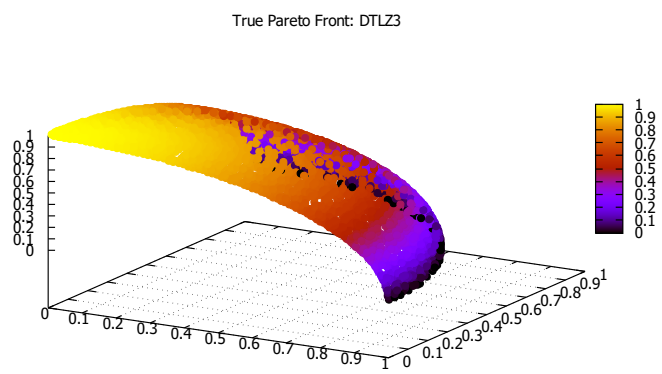

(a) True Pareto Front

HRISE_M: DTLZ3

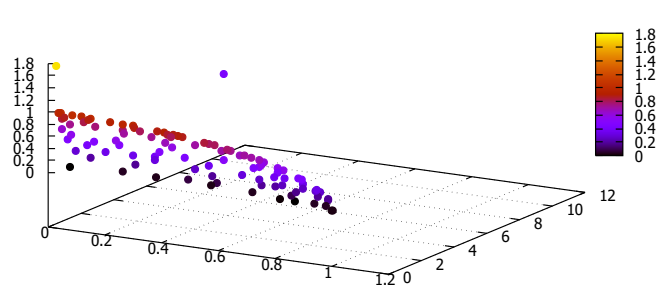

(c) Solution Front: HRISE_M

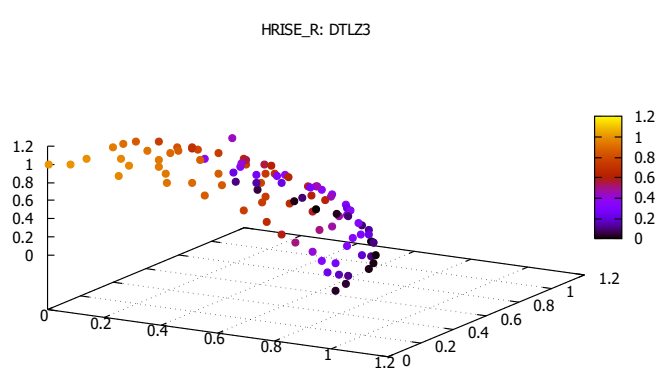

(b) Solution Front: HRISE_R

HH-RILA: DTLZ3

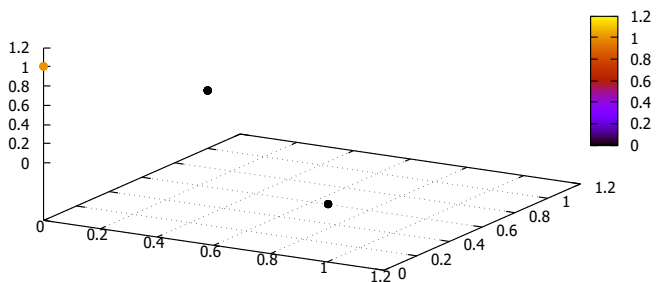

(d) Solution Front: HH-RILA

Figure 9: One of the best performances of our approaches: DTLZ3 (benchmark)

Regarding the real-world problem instances, Figures 11 and 12 show one of the best and worst performances, respectively, compared to HH-RILA. It is evident that in SP1 (three objectives) our approaches surpass HH-RILA and the opposite happens in VC3 (two objectives).

Finally, we would like to emphasise the diversity of different problem instances, unconstrained and constrained, that we addressed in the evaluation. As we know, there is a huge interest from the community in assessing complicated real-world problems [52]. We believe we approached this

\footnotetext{
${ }^{3}$ The final population obtained by the algorithms $\left(P_{o p}{ }^{f}\right.$ in Algorithm 1$)$ is also known as the Solution Set. The Solution Front lies in the objective space, and it is composed of the values of the objective functions of the elements (solutions) in the Solution Set.
} 


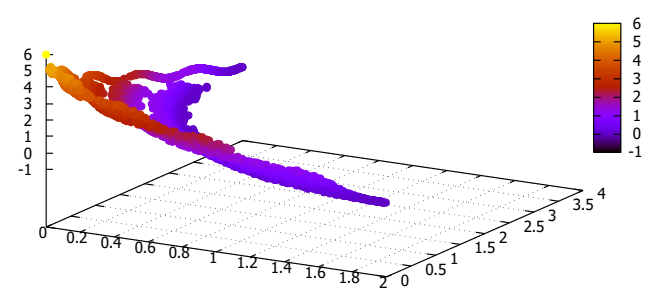

(a) True Pareto Front

HRISE_M: WFG1

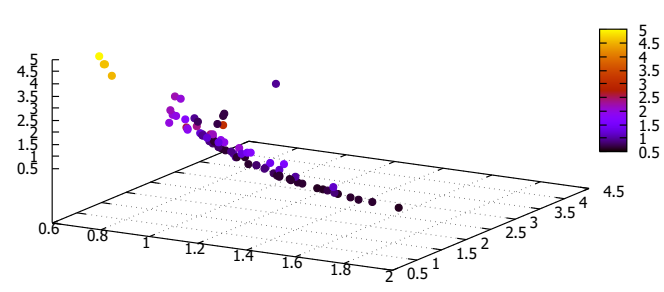

(c) Solution Front: HRISE_M

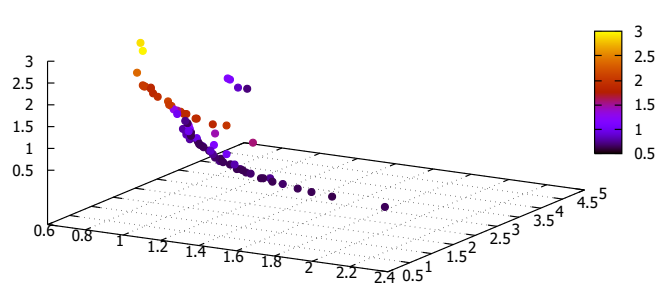

(b) Solution Front: HRISE_R

Figure 10: One of the worst performances of our approaches: WFG1 (benchmark)

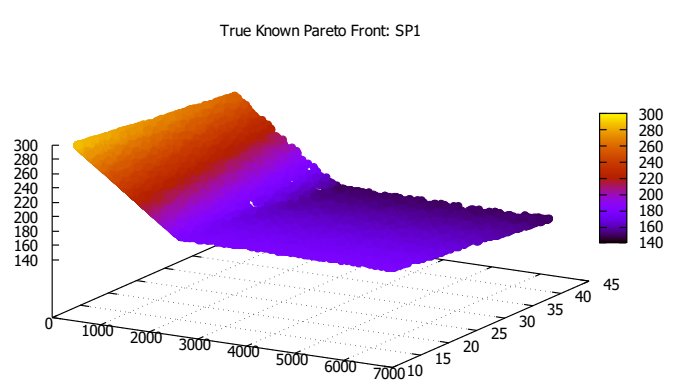

(a) True Known Pareto Front

HRISE_M: SP1

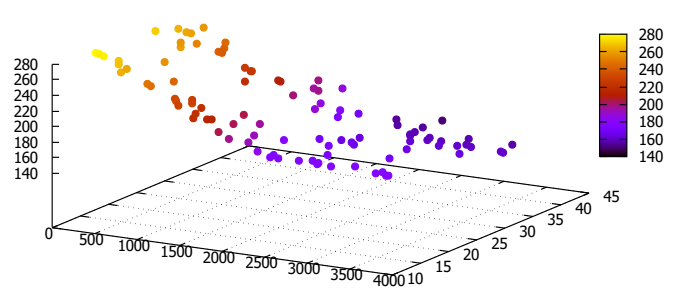

(c) Solution Front: HRISE_M

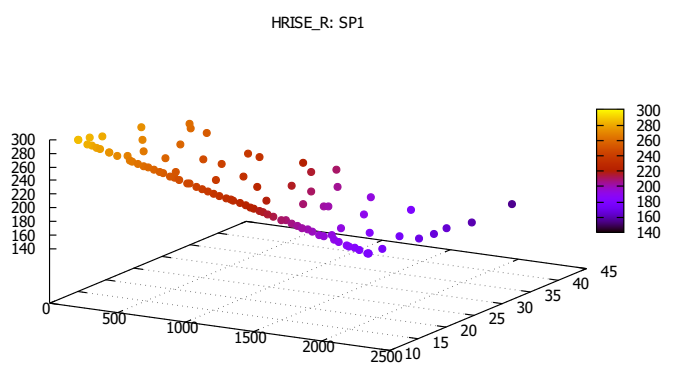

(b) Solution Front: HRISE_R

HH-RILA: SP1

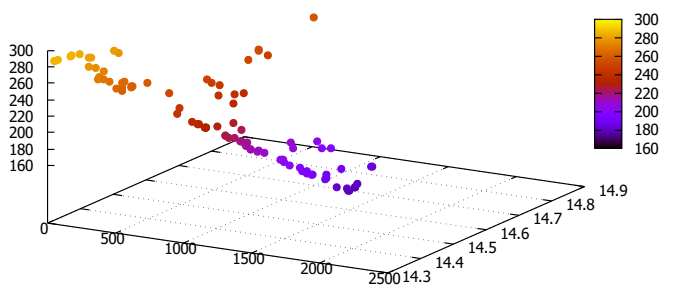

(d) Solution Front: HH-RILA

Figure 11: One of the best performances of our approaches: SP1 (real) 


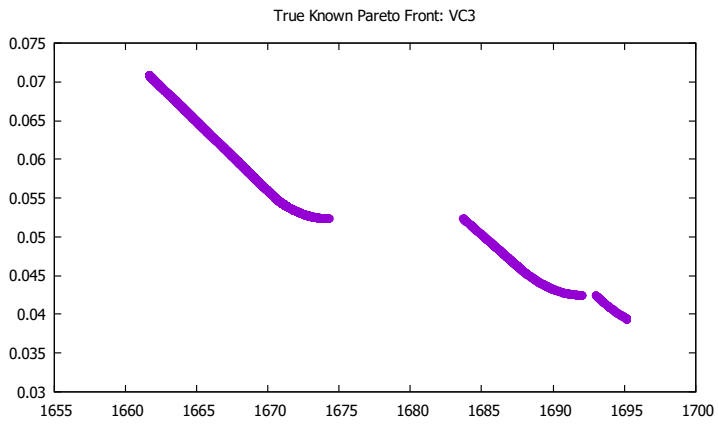

(a) True Known Pareto Front

HRISE_M: VC3

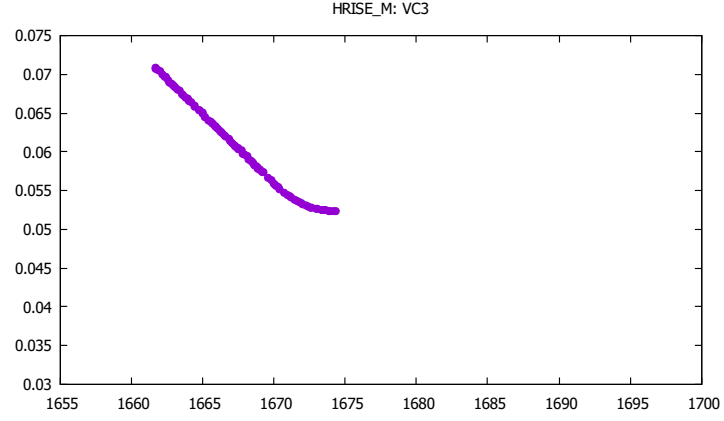

(c) Solution Front: HRISE_M

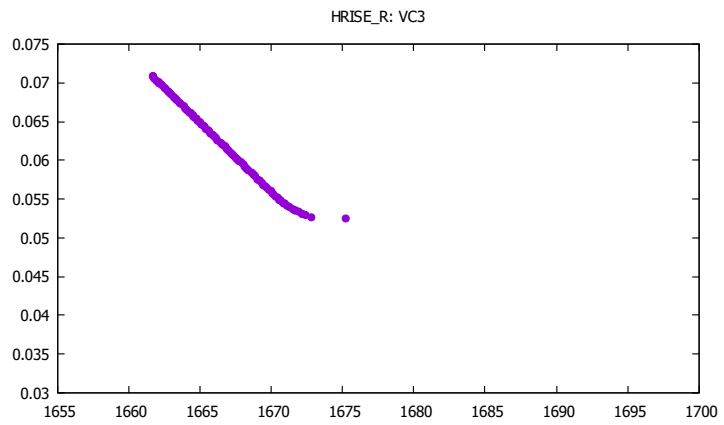

(b) Solution Front: HRISE_R

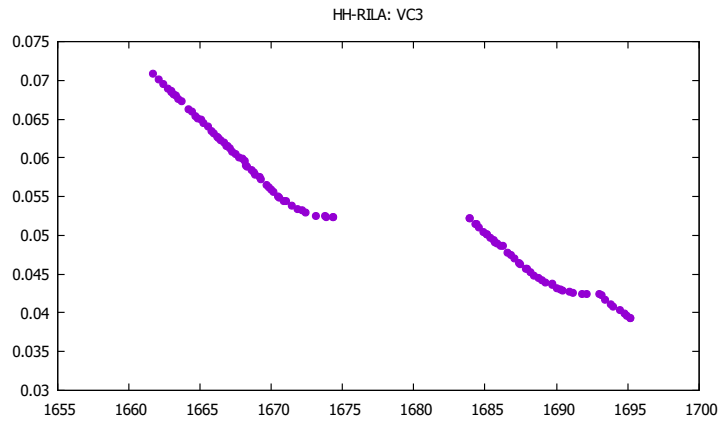

(d) Solution Front: HH-RILA

Figure 12: One of the worst performances of our approaches: VC3 (real)

five objective functions. To highlight this point, we show the True Known Pareto Front of the real-world problem instance VC1 in Figure 13 Looking at this front, we see that there is no clear pattern or characteristic that defines it and, moreover, it presents discontinuities posing difficulties for the algorithms to solve such problem instance.

True Known Pareto Front: VC1

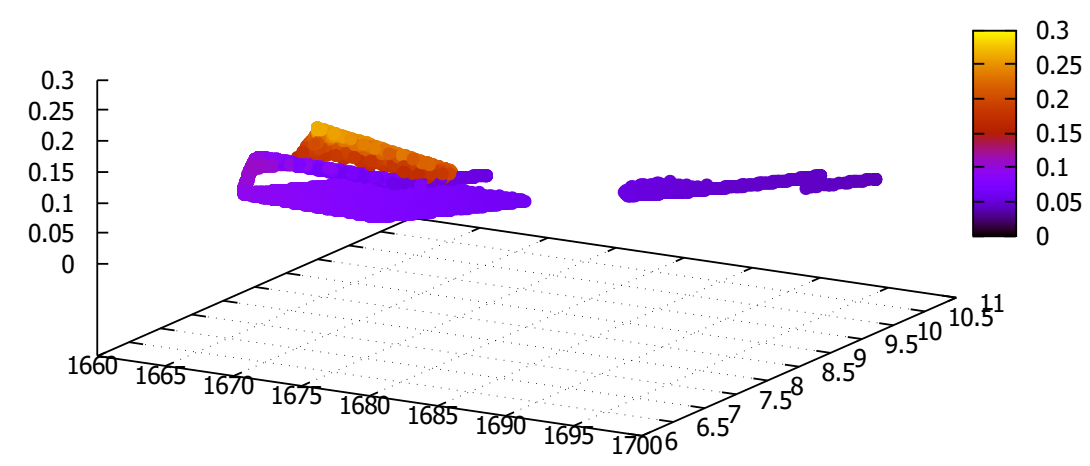

Figure 13: True Known Pareto Front: VC1 (real) 

with a few medium positive relationships. On the other hand, HRMA had mostly weak correlation with even a negative one in the VC problem. Figure 14 shows the performance of HRISE_R and HRMA for the VC problem.

Table 8: Correlation analysis: selected LLHs $\times$ accepted populations

\begin{tabular}{c|ccc}
\hline Problem & Algorithm & $\tau$ & Conclusion \\
\hline \hline DTLZ & & & \\
\hline & HRISE_R & 0.704 & strong+ \\
& HRISE_M & 0.66 & strong+ \\
& HRMA & 0.133 & weak+ \\
\hline WFG & & & \\
\hline & HRISE_R & 0.799 & strong+ \\
& HRISE_M & 0.754 & strong+ \\
& HRMA & 0.383 & medium+ \\
\hline VC & & & \\
\hline & HRISE_R & 0.779 & strong+ \\
& HRISE_M & 0.646 & strong+ \\
& HRMA & -0.156 & weak- \\
\hline WR & & & \\
\hline & HRISE_R & 0.475 & medium+ \\
& HRISE_M & 0.654 & strong+ \\
& HRMA & 0.262 & weak+ \\
\hline CS & & & \\
\hline & HRISE_R & 0.706 & strong+ \\
& HRISE_M & 0.366 & medium+ \\
& HRMA & -0.114 & no \\
\hline & HRISE_R & 0.514 & strong+ \\
& HRISE_M & 0.725 & strong+ \\
& wrMA & 0.171 & weak+ \\
\hline
\end{tabular}
matters and are more suitable than a simple random approach. 


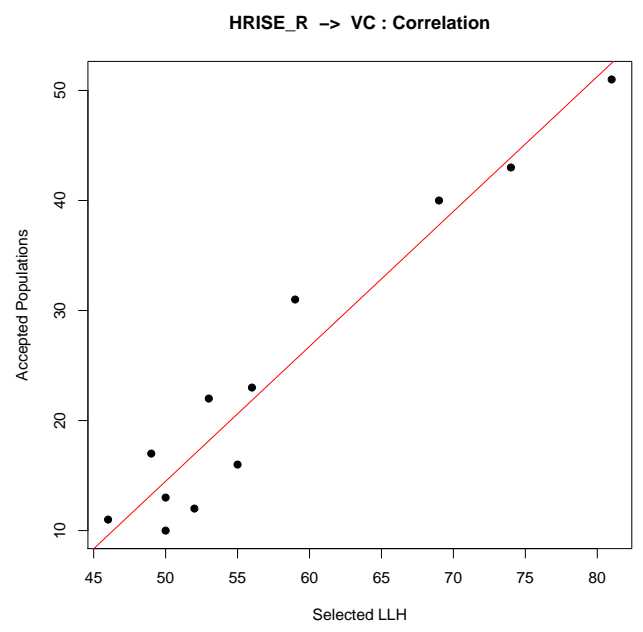

(a) HRISE_R

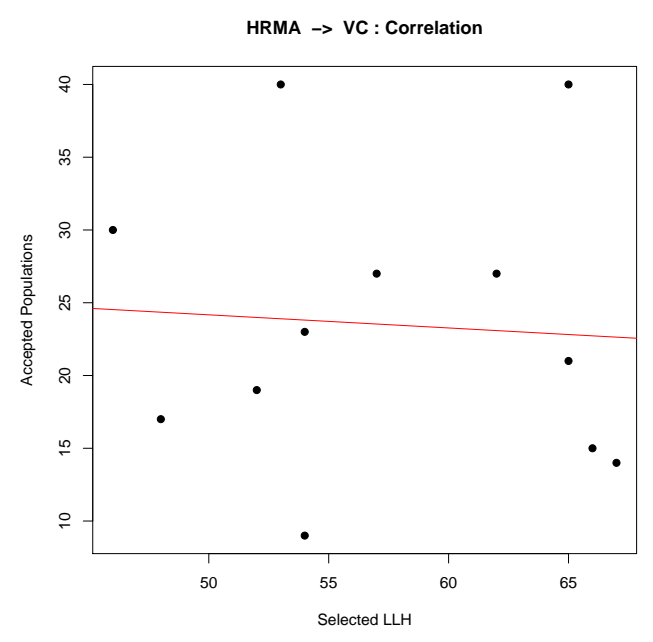

(b) HRMA

Figure 14: Correlation between selected LLHs and accepted populations: VC (real)

\section{Conclusions}

While the studies on selection hyper-heuristics have rapidly been increasing based on single point-based search for single objective optimisation, the studies on multi-objective hyper-heuristics

are still missing in the scientific literature, possibly because it is not straightforward to design a hyper-heuristic combining an appropriate heuristic selection method with a robust acceptance method, and the use of population-based approaches as low-level (meta)heuristics introduces additional complexities.

In this study, we presented the HRISE selection hyper-heuristics for multi-objective optimisation managing a set of low-level MOEAs and embedding the following novel algorithmic components:

1. Reinforcement Learning-based metaheuristic selection method complemented by a balanced exploitation/exploration technique;

2. Dynamic control mechanism (nonuniform iterations strategy) deciding how long (for how many iterations) a selected LLH will run;

3. Two-level (hierarchical) acceptance approach, Only Improving and group decision-making, where in the latter we have the responsibility and majority rules (denoted as hyper-heuristics HRISE_R and HRISE_M, respectively); and

4. QLA and MQLA acceptance methods proposed to be used within the group along with GDA. 
A third proposed hyper-heuristic is HRMA where LLH selection is random but we still have the nonuniform strategy, and we make a random selection of all available move acceptance methods. Previous works in selection hyper-heuristics for single objective optimisation show the importance of learning in heuristic selection [67] as well as how crucial the choice of move acceptance is multi-objective optimisation and additionally emphasises that an improved overall performance is possible using multiple acceptance methods under a group decision-making scheme.

The empirical results, across 39 problem instances from four classes of benchmark functions and four real-world problems, where we evaluated both unconstrained and constrained problems,

illustrate that our hyper-heuristics, with the proposed algorithmic components, performed the best when compared to HH-CF, HH-ALL, and each low-level MOEA run in isolation in terms of hypervolume. Still regarding the hypervolume, both HRISE hyper-heuristics and HRMA deliver a slightly better performance than the recently proposed state-of-the-art selection hyper-heuristic HH-RILA. Although this performance variation is not statistically significant, we may conclude that, considering the problem instances we selected, our hyper-heuristics were better than $\mathrm{HH}$ RILA taking into account the pairwise comparison between HH-RILA and some other approaches where HH-RILA was not better statistically speaking but our hyper-heuristics were. Considering the $\epsilon$ indicator, HRISE_R was the best followed by HH-RILA. Overall, considering both quality indicators, HRISE_R presented the best performance of all hyper-heuristics.

A natural future research direction would be evaluating and analysing the whole approach under various configurations, for example, including different set of low-level metaheuristics and/or adding more move acceptance methods, such as the Iteration Limited Threshold Accepting (ILTA) 68] and Adaptive Simulated Annealing (ASA) [69] for multi-objective optimisation. Additionally, the proposed framework can be evaluated for many-objective optimisation based on other relevant metrics, such as fast computation hypervolume [70] along with the RPO indicator proposed in this paper, and relying on other relevant approaches as low-level metaheuristics, such as NSGA-III [71] and the Many Objective Metaheuristic Based on the R2 indicator-II (MOMBI-II) 72. Another natural extension to the current work would be applying this general-purpose approach to several additional unseen real-world multi/many-objective problems to evaluate its performance, aiming to conclude definitively in terms of generalisation.

\section{Acknowledgements}

This research was supported by Fundação de Amparo à Pesquisa do Estado de São Paulo (FAPESP), Brazil, process number: 2018/08372-8. 


\section{References}

760 [1] E. K. Burke, M. R. Hyde, G. Kendall, G. Ochoa, E. Özcan, J. R. Woodward, A classification of hyper-heuristic approaches: Revisited, in: M. Gendreau, J.-Y. Potvin (Eds.), Handbook of

\ Metaheuristics, Springer International Publishing, Cham, 2019, pp. 453-477. doi:10.1007/ 978-3-319-91086-4_14.

URL https://doi.org/10.1007/978-3-319-91086-4_14

${ }_{765}^{\text {口 }}$ [2] E. K. Burke, M. Gendreau, M. Hyde, G. Kendall, G. Ochoa, E. Özcan, R. Qu, Hyperheuristics: A survey of the state of the art, Journal of the Operational Research Society 64 (12) (2013) 1695-1724. doi:10.1057/jors.2013.71.

URL https://doi.org/10.1057/jors.2013.71

口 [3] E. Özcan, M. Misir, G. Ochoa, E. K. Burke, A reinforcement learning-great-deluge hyperheuristic for examination timetabling, Int. J. Appl. Metaheuristic Comput. 1 (1) (2010) 39-59. doi:10.4018/jamc.2010102603. URL http://dx.doi.org/10.4018/jamc. 2010102603

[4] P. Cowling, G. Kendall, E. Soubeiga, A hyperheuristic approach to scheduling a sales summit, in: E. Burke, W. Erben (Eds.), Practice and Theory of Automated Timetabling III, Springer Berlin Heidelberg, Berlin, Heidelberg, 2001, pp. 176-190.

[5] P. Cowling, G. Kendall, E. Soubeiga, Hyperheuristics: A tool for rapid prototyping in scheduling and optimisation, in: S. Cagnoni, J. Gottlieb, E. Hart, M. Middendorf, G. R. Raidl (Eds.), Applications of Evolutionary Computing, Springer Berlin Heidelberg, Berlin, Heidelberg, 2002, pp. 1-10.

[6] M. Ayob, G. Kendall, A Monte Carlo hyper-heuristic to optimise component placement sequencing for multi head placement machine, in: Placement Machine, INTECH'03 Thailand, 2003, pp. 132-141.

[7] D. Pisinger, S. Ropke, A general heuristic for vehicle routing problems, Computers \& Opera1. tions Research 34 (8) (2007) 2403 - 2435. doi:https://doi.org/10.1016/j.cor.2005.09. $785 \quad 012$.

URL http://www.sciencedirect.com/science/article/pii/S0305054805003023

[8] E. Ozcan, Y. Bykov, M. Birben, E. K. Burke, Examination timetabling using late acceptance hyper-heuristics, in: 2009 IEEE Congress on Evolutionary Computation, 2009, pp. 997-1004. doi:10.1109/CEC.2009.4983054. 
[9] J. H. Drake, A. Kheiri, E. Özcan, E. K. Burke, Recent advances in selection hyper-heuristics, European Journal of Operational Research 285 (2) (2020) 405-428. doi:https://doi.org/ 10.1016/j.ejor.2019.07.073. URL http://www.sciencedirect.com/science/article/pii/S0377221719306526

[10] G. Guizzo, S. R. Vergilio, A. T. R. Pozo, G. M. Fritsche, A multi-objective and evolutionary hyper-heuristic applied to the integration and test order problem, Applied Soft Computing 56 (2017) 331 - 344. doi:https://doi.org/10.1016/j.asoc.2017.03.012. URL http://www.sciencedirect.com/science/article/pii/S1568494617301357

[11] T. N. Ferreira, J. A. P. Lima, A. Strickler, J. N. Kuk, S. R. Vergilio, A. Pozo, Hyper-heuristic based product selection for software product line testing, IEEE Computational Intelligence Magazine 12 (2) (2017) 34-45. doi:10.1109/MCI.2017.2670461.

[12] J. A. P. Lima, S. R. Vergilio, A multi-objective optimization approach for selection of second order mutant generation strategies, in: Proceedings of the 2nd Brazilian Symposium on Systematic and Automated Software Testing, SAST, ACM, New York, NY, USA, 2017, pp. 6:1-6:10. doi:10.1145/3128473.3128479. URL http://doi.acm.org/10.1145/3128473.3128479

[13] M. Maashi, E. Özcan, G. Kendall, A multi-objective hyper-heuristic based on choice function, Expert Systems with Applications 41 (9) (2014) 4475 - 4493. doi:https://doi.org/10. $1016 / \mathrm{j}$.eswa.2013.12.050 URL http://www.sciencedirect.com/science/article/pii/S095741741400013X

[14] W. Li, E. Özcan, R. John, Multi-objective evolutionary algorithms and hyper-heuristics for wind farm layout optimisation, Renewable Energy 105 (2017) 473 - 482. doi:https://doi. org $/ 10.1016 /$ j.renene.2016.12.022 URL http://www.sciencedirect.com/science/article/pii/S0960148116310709

[15] V. R. de Carvalho, K. Larson, A. A. F. Brandão, J. S. Sichman, Applying social choice theory to solve engineering multi-objective optimization problems, Journal of Control, Automation and Electrical Systems 31 (2020) 119-128. doi:10.1007/s40313-019-00526-2.

[16] W. Li, E. Özcan, R. John, A learning automata-based multiobjective hyper-heuristic, IEEE घ Transactions on Evolutionary Computation 23 (1) (2019) 59-73. doi:10.1109/TEVC.2017. 2785346 .

[17] A. Kheiri, M. Misır, E. Özcan, Ensemble move acceptance in selection hyper-heuristics, in: T. Czachórski, E. Gelenbe, K. Grochla, R. Lent (Eds.), Computer and Information Sciences, Springer International Publishing, Cham, 2016, pp. 21-29. 
[18] K. Deb, A. Pratap, S. Agarwal, T. Meyarivan, A fast and elitist multiobjective genetic algorithm: NSGA-II, IEEE Transactions on Evolutionary Computation 6 (2) (2002) 182-197.

[26] A. Saeed, S. H. A. Hamid, M. B. Mustafa, The experimental applications of search-based techniques for model-based testing: Taxonomy and systematic literature review, Applied Soft Computing 49 (2016) 1094 - 1117. doi:https://doi.org/10.1016/j.asoc.2016.08.030. URL http://www.sciencedirect.com/science/article/pii/S1568494616304240 
[27] J. M. Balera, V. A. Santiago Júnior, A systematic mapping addressing hyper-heuristics within search-based software testing, Information and Software Technology 114 (2019) 176 - 189. doi:https://doi.org/10.1016/j.infsof.2019.06.012 URL http://www.sciencedirect.com/science/article/pii/S0950584919301430

[28] M. Khari, P. Kumar, An extensive evaluation of search-based software testing: A review, Soft Computing 23 (6) (2019) 1933-1946. doi:10.1007/s00500-017-2906-y. URL https://doi.org/10.1007/s00500-017-2906-y

[29] J.-Y. Audibert, R. Munos, C. Szepesvári, Exploration-exploitation tradeoff using variance estimates in multi-armed bandits, Theoretical Computer Science 410 (19) (2009) 1876 - 1902, algorithmic Learning Theory. doi:https://doi.org/10.1016/j.tcs.2009.01.016. URL http://www.sciencedirect.com/science/article/pii/S030439750900067X

[30] S. Jain, S. Gujar, S. Bhat, O. Zoeter, Y. Narahari, A quality assuring, cost optimal multiarmed bandit mechanism for expertsourcing, Artificial Intelligence 254 (2018) 44 - 63. doi: https://doi.org/10.1016/j.artint.2017.10.001. URL http://www.sciencedirect.com/science/article/pii/S000437021730125X

[31] C. M. Fonseca, P. J. Fleming, Multiobjective optimization and multiple constraint handling with evolutionary algorithms. I. A unified formulation, IEEE Transactions on Systems, Man, and Cybernetics - Part A: Systems and Humans 28 (1) (1998) 26-37. doi:10.1109/3468. 650319

[32] G. Dueck, New optimization heuristics: The great deluge algorithm and the record-to-record travel, Journal of Computational Physics 104 (1) (1993) 86 - 92. doi:https://doi.org/10. 1006/jcph.1993.1010

URL http://www.sciencedirect.com/science/article/pii/S0021999183710107

[33] S. Kukkonen, J. Lampinen, GDE3: The third evolution step of generalized differential evolution, in: 2005 IEEE Congress on Evolutionary Computation, Vol. 1, 2005, pp. 443-450 Vol.1. doi:10.1109/CEC.2005.1554717.

[34] E. Shojaedini, M. Majd, R. Safabakhsh, Novel adaptive genetic algorithm sample consensus 1. Applied Soft Computing 77 (2019) 635 - 642. doi:https://doi.org/10.1016/j.asoc. 2019.01 .052

URL http://www.sciencedirect.com/science/article/pii/S1568494619300651

[35] E. Zitzler, L. Thiele, Multiobjective evolutionary algorithms: A comparative case study and the strength pareto approach, IEEE Transactions on Evolutionary Computation 3 (4) (1999) 257-271. doi:10.1109/4235.797969 
[36] K. Bringmann, T. Friedrich, The maximum hypervolume set yields near-optimal approximation, in: Proceedings of the 12th Annual Conference on Genetic and Evolutionary Computation, GECCO '10, ACM, New York, NY, USA, 2010, pp. 511-518. doi:10.1145/1830483. 1830576 .

URL http://doi .acm.org/10.1145/1830483.1830576

[37] S. Jiang, Y. Ong, J. Zhang, L. Feng, Consistencies and contradictions of performance metrics in multiobjective optimization, IEEE Transactions on Cybernetics 44 (12) (2014) 2391-2404. doi:10.1109/TCYB.2014.2307319.

[38] D. A. Van Veldhuizen, G. B. Lamont, On measuring multiobjective evolutionary algorithm performance, in: Proceedings of the 2000 Congress on Evolutionary Computation. CEC00 (Cat. No.00TH8512), Vol. 1, 2000, pp. 204-211 vol.1. doi:10.1109/CEC.2000.870296.

${ }_{[}[39]$ K. Tan, T. Lee, E. Khor, Evolutionary algorithms for multi-objective optimization: Performance assessments and comparisons, Artificial Intelligence Review 17 (4) (2002) 251-290. doi:10.1023/A:1015516501242. URL https://doi.org/10.1023/A:1015516501242

[40] D. A. Van Veldhuizen, G. B. Lamont, Multiobjective evolutionary algorithm test suites, in: Proceedings of the 1999 ACM Symposium on Applied Computing, SAC '99, ACM, New York, NY, USA, 1999, pp. 351-357. doi:10.1145/298151.298382.

URL http://doi .acm.org/10.1145/298151.298382

[41] E. Zitzler, L. Thiele, M. Laumanns, C. M. Fonseca, V. G. da Fonseca, Performance assessment of multiobjective optimizers: An analysis and review, IEEE Transactions on Evolutionary Computation 7 (2) (2003) 117-132. doi:10.1109/TEVC.2003.810758.

[42] J. R. Schott, Fault tolerant design using single and multicriteria genetic algorithm optimization, Ph.D. thesis, Massachusetts Institute of Technology (MIT), Dept. of Aeronautics and Astronautics (1995).

[43] H. Li, Q. Zhang, Multiobjective optimization problems with complicated pareto sets, MOEA/D and NSGA-II, IEEE Transactions on Evolutionary Computation 13 (2) (2009) 284-302. doi:10.1109/TEVC.2008.925798.

[44] H. Ishibuchi, H. Masuda, Y. Nojima, A study on performance evaluation ability of a modified inverted generational distance indicator, in: Proceedings of the 2015 Annual Conference on Genetic and Evolutionary Computation, GECCO '15, ACM, New York, NY, USA, 2015, pp. 695-702. doi:10.1145/2739480.2754792. 
[45] A. Zhou, Y. Jin, Q. Zhang, B. Sendhoff, E. Tsang, Combining model-based and geneticsbased offspring generation for multi-objective optimization using a convergence criterion, in: 2006 IEEE International Conference on Evolutionary Computation, 2006, pp. 892-899. doi:10.1109/CEC.2006.1688406.

925

[52] Q. Zhang, A. Zhou, S. Zhao, P. N. Suganthan, W. Liu, S. Tiwari, Multiobjective optimization test instances for the CEC 2009 Special Session and Competition, Available from: https://bit.ly/35M9818, Access in: Jan 7, 2020. (2009).

[53] X. Liao, Q. Li, X. Yang, W. Zhang, W. Li, Multiobjective optimization for crash safety design of vehicles using stepwise regression model, Structural and Multidisciplinary Optimization 35 (6) (2008) 561-569. doi:10.1007/s00158-007-0163-x

URL https://doi.org/10.1007/s00158-007-0163-x 
[54] D. P. Loucks, E. van Beek, Water resources planning and management: An overview, Springer International Publishing, Cham, 2017, pp. 1-49. doi:10.1007/978-3-319-44234-1_1. URL https : //doi .org/10.1007/978-3-319-44234-1_1

[55] T. Ray, K. Tai, K. C. Seow, Multiobjective design optimization by an evolutionary algo-

1. rithm, Engineering Optimization 33 (4) (2001) 399-424. arXiv:https://doi.org/10.1080/ 03052150108940926 , doi:10.1080/03052150108940926.

URL https ://doi .org/10.1080/03052150108940926

[56] A. Sinha, D. K. Saxena, K. Deb, A. Tiwari, Using objective reduction and interactive procedure to handle many-objective optimization problems, Appl. Soft Comput. 13 (1) (2013) 415-427. doi:10.1016/j.asoc.2012.08.030

URL http://dx.doi.org/10.1016/j.asoc.2012.08.030

[57] V. A. Santiago Júnior, S. Tahar, Time performance formal evaluation of complex systems, in: M. Cornélio, B. Roscoe (Eds.), Formal Methods: Foundations and Applications, Springer International Publishing, Cham, 2016, pp. 162-177.

[58] J. Braga, F. D’Amico, M. A. C. Avila, A. V. Penacchioni, J. R. Sacahui, V. A. Santiago Júnior, F. Mattiello-Francisco, C. Strauss, M. A. A. Fialho, The protoMIRAX hard x-ray imaging balloon experiment, Astronomy \& Astrophysics 580 (2015) A108.

[59] J. J. Durillo, A. J. Nebro, jMetal: A java framework for multi-objective optimization, Ad口 vances in Engineering Software 42 (10) (2011) 760 - 771. doi:https://doi.org/10.1016/ j.advengsoft.2011.05.014

URL http://www.sciencedirect.com/science/article/pii/S0965997811001219

[60] V. R. de Carvalho, jMetalHyperHeuristicHelper web site, Available from: https://bit.ly/2G11gyx, Access in: Aug 07, 2019.

[61] K. Deb, R. B. Agrawal, Simulated binary crossover for continuous search space, Complex Systems 9 (2) (1995) 115-148.

[62] M. Wagner, K. Bringmann, T. Friedrich, F. Neumann, Efficient optimization of many objectives by approximation-guided evolution, European Journal of Operational Research 243 (2) (2015) 465 - 479. doi:https://doi.org/10.1016/j.ejor.2014.11.032. URL http://www.sciencedirect.com/science/article/pii/S0377221714009552

[63] V. A. Santiago Júnior, E. Özcan, V. R. de Carvalho, Experimental evaluation data: Applied Soft Computing 2020, available from: https://bit.ly/2kuPAgc. Access in: Jan 12, 2020. (2020). 
985 [64] T. Hothorn, K. Hornik, M. van de Wiel, A. Zeileis, Implementing a class of permutation tests:

口. The coin package, Journal of Statistical Software 28 (8) (2008) 1-23. doi:10.18637/jss. v028.i08.

URL https://www.jstatsoft.org/v028/i08

[65] J. M. Balera, V. A. Santiago Júnior, An algorithm for combinatorial interaction testing: definitions and rigorous evaluations, Journal of Software Engineering Research and Development 5 (1) (2017) 10. doi:10.1186/s40411-017-0043-z

URL https://doi.org/10.1186/s40411-017-0043-z

[66] L. B. R. Santos, V. A. Santiago Júnior, L. V. Povoa, A. V. Freitas, C. C. Mario, Software inspections: comparing a formal method based with a classical reading methodology, International Journal of Computer Applications in Technology 59 (4) (2019) 296 - 317. doi:10.1504/IJCAT .2019.099198.

[67] E. Özcan, B. Bilgin, E. E. Korkmaz, A comprehensive analysis of hyper-heuristics, Intell. Data Anal. 12 (1) (2008) 3-23.

URL http://dl .acm.org/citation. cfm?id=1368027.1368029

[68] M. Misir, T. Wauters, K. Verbeeck, G. V. Berghe, A new learning hyper-heuristic for the traveling tournament problem, in: Proceedings of the 8th Metaheuristic International Conference (MIC'09), 2009, pp. id1-id10.

[69] X. Geng, Z. Chen, W. Yang, D. Shi, K. Zhao, Solving the traveling salesman problem based on an adaptive simulated annealing algorithm with greedy search, Applied Soft Computing
11 (4) (2011) 3680 - 3689. doi:https://doi.org/10.1016/j.asoc.2011.01.039.

URL http://www.sciencedirect.com/science/article/pii/S1568494611000573

[70] S. Rostami, F. Neri, A fast hypervolume driven selection mechanism for many-objective

1. optimisation problems, Swarm and Evolutionary Computation 34 (2017) 50 - 67. doi: https : //doi.org/10.1016/j.swevo.2016.12.002

URL http://www.sciencedirect.com/science/article/pii/S2210650216301328

[71] K. Deb, H. Jain, An evolutionary many-objective optimization algorithm using referencepoint-based nondominated sorting approach, Part I: Solving problems with box constraints, IEEE Transactions on Evolutionary Computation 18 (4) (2014) 577-601. doi:10.1109/TEVC. 2013.2281535

[72] R. H. Gómez, C. A. C. Coello, Improved metaheuristic based on the R2 indicator for manyobjective optimization, in: Proceedings of the 2015 Annual Conference on Genetic and Evolutionary Computation, GECCO '15, ACM, New York, NY, USA, 2015, pp. 679-686. 
doi:10.1145/2739480.2754776.

URL http://doi .acm.org/10.1145/2739480.2754776 